\title{
The genome of the yellow potato cyst nematode, Globodera rostochiensis, reveals insights into the basis of parasitism and virulence
}

Sebastian Eves-van den Akker ${ }^{1 *+}$, Dominik R. Laetsch ${ }^{2 \dagger}$, Peter Thorpe ${ }^{3+}$, Catherine J. Lilley ${ }^{4 \dagger}$, Etienne G. J. Danchin ${ }^{5}$, Martine Da Rocha ${ }^{5}$, Corinne Rancurel ${ }^{5}$, Nancy E. Holroyd ${ }^{6}$, James A. Cotton ${ }^{6}$, Amir Szitenberg ${ }^{7}$, Eric Grenier ${ }^{8}$, Josselin Montarry ${ }^{8}$, Benjamin Mimee ${ }^{9}$, Marc-Olivier Duceppe ${ }^{9}$, Ian Boyes ${ }^{10}$, Jessica M. C. Marvin ${ }^{4}$, Laura M. Jones ${ }^{4}$, Hazijah B. Yusup ${ }^{4}$, Joël Lafond-Lapalme ${ }^{9}$, Magali Esquibet ${ }^{8}$, Michael Sabeh ${ }^{9}$, Michael Rott ${ }^{10}$, Hein Overmars ${ }^{11}$, Anna Finkers-Tomczak ${ }^{11}$, Geert Smant ${ }^{11}$, Georgios Koutsovoulos ${ }^{2}$, Vivian Blok ${ }^{3}$, Sophie Mantelin ${ }^{3}$, Peter J. A. Cock ${ }^{12}$, Wendy Phillips ${ }^{13}$, Bernard Henrissat ${ }^{14,15}$, Peter E. Urwin ${ }^{4}$, Mark Blaxter ${ }^{2}$ and John T. Jones 3,16

\begin{abstract}
Background: The yellow potato cyst nematode, Globodera rostochiensis, is a devastating plant pathogen of global economic importance. This biotrophic parasite secretes effectors from pharyngeal glands, some of which were acquired by horizontal gene transfer, to manipulate host processes and promote parasitism. G. rostochiensis is classified into pathotypes with different plant resistance-breaking phenotypes.

Results: We generate a high quality genome assembly for G. rostochiensis pathotype Ro1, identify putative effectors and horizontal gene transfer events, map gene expression through the life cycle focusing on key parasitic transitions and sequence the genomes of eight populations including four additional pathotypes to identify variation. Horizontal gene transfer contributes $3.5 \%$ of the predicted genes, of which approximately $8.5 \%$ are deployed as effectors. Over one-third of all effector genes are clustered in 21 putative 'effector islands' in the genome. We identify a dorsal gland promoter element motif (termed DOG Box) present upstream in representatives from 26 out of 28 dorsal gland effector families, and predict a putative effector superset associated with this motif. We validate gland cell expression in two novel genes by in situ hybridisation and catalogue dorsal gland promoter element-containing effectors from available cyst nematode genomes. Comparison of effector diversity between pathotypes highlights correlation with plant resistance-breaking.
\end{abstract}

Conclusions: These G. rostochiensis genome resources will facilitate major advances in understanding nematode plant-parasitism. Dorsal gland promoter element-containing effectors are at the front line of the evolutionary arms race between plant and parasite and the ability to predict gland cell expression a priori promises rapid advances in understanding their roles and mechanisms of action.

Keywords: Plant-parasitic nematode, Genome sequence, Virulence, Effectors, Horizontal gene transfer

\footnotetext{
* Correspondence: s.evesvandenakker@dundee.ac.uk

${ }^{\dagger}$ Equal contributors

SEVDA and DRL contributed equally

PT and $C J$ contributed equally

'Division of Plant Sciences, College of Life Sciences, University of Dundee,

Dundee DD1 5EH, UK

Full list of author information is available at the end of the article
} 


\section{Background}

All major crops are thought to be infected by at least one species of plant-parasitic nematode, which causes damage valued at over $\$ 80$ billion each year [1]. The majority of these economic losses are attributable to the sedentary endoparasitic nematodes of the genus Meloidogyne (root-knot nematodes) and the genera Heterodera and Globodera (cyst nematodes). These sedentary endoparasites have complex biotrophic interactions with their hosts that include induction of specific feeding sites and long residence times within or on their host(s).

Potato cyst nematodes (PCN) are economically important pathogens of potato, with two major species: the white PCN Globodera pallida and the yellow PCN G. rostochiensis. These nematodes originate in South America $[2,3]$ and have subsequently been introduced into all major potato-growing regions of the world. Europe has acted as a secondary distribution hub for PCN; worldwide populations outside South America reflect subsequent introductions from Europe [4, 5]. Once established in a field, PCN are effectively impossible to eradicate in the short term and because they persist as long-lived cysts in soils, growing potatoes may not be economically viable for up to two decades. As a result, the US Department of Agriculture (USDA) has classified the yellow PCN as potentially more dangerous than any insect or disease affecting the potato industry (Aphis USDA 12/09/2015). Substantial effort is thus invested into keeping land free of $\mathrm{PCN}$; both species are present on USDA and European Plant Protection Organisation quarantine organism lists.

PCN have been classified to pathotype based on their relative virulence on host plants harbouring different resistance loci. Most of the G. rostochiensis in UK potato-growing regions is of pathotype Rol and can be controlled by a single major resistance locus (H1). UK G. rostochiensis populations have therefore been suggested to originate from a genetically restricted introduction into Europe [6, 7]. Other pre-existing G. rostochiensis pathotypes (Ro 2, 3 and 5, but not 4) are able to overcome $\mathrm{H} 1$ resistance [8] and these pathotypes may be selected in response to widespread deployment of $\mathrm{H} 1$ plants. The corresponding nematode avirulence gene(s) has not been identified. Understanding the bases of virulence and resistance is of critical importance for agriculture.

G. rostochiensis has a complex life cycle that includes a highly resistant survival stage. Cysts, formed from the body wall of the adult female, encase hundreds of eggs that can lie dormant in the soil for over 20 years. Second stage juveniles (J2) within the eggs hatch in response to root diffusates from suitable host plants growing nearby. The $\mathrm{J} 2$ nematodes locate the root and migrate destructively through root tissues until they reach the inner cortex layers. Here the nematodes probe the cells, until a cell that does not respond adversely is detected [9]. This initial syncytial cell is transformed into a large, multinucleate syncytium in response to proteins, peptides and hormones secreted by the nematode. Cell wall openings are formed between the initial syncytial cell and its neighbours, followed by fusion of the protoplasts. Syncytial cells become highly metabolically active and have enriched cytoplasm, enlarged nuclei and a greatly reduced central vacuole. Additional layers of cells are subsequently incorporated into the syncytium, which may eventually be composed of up to 300 cells [9]. A prolonged biotrophic interaction is then maintained for a period of several weeks, while the nematode intermittently withdraws host cytoplasm to derive all food required for development to the adult stage. Each nematode can only induce a single feeding site that must therefore be maintained and protected from host defences.

The complex interactions of PCN with their hosts, like those of other plant parasites and pathogens, are mediated by effectors: secreted proteins that manipulate the host to the benefit of the pathogen. Most PCN effectors are produced in two sets of gland cells, dorsal and subventral [10], although some apoplastic effectors can be produced in the gland cells surrounding the main anterior sensory organs, the amphids [11]. Effectors play important roles in all aspects of the parasite-host interaction: invasion and migration [12], suppression of host defences [13] and induction of the feeding site [14, 15]. The effector repertoire of plant-parasitic nematodes, including PCN, has been augmented by multiple Horizontal Gene Transfer (HGT) events, primarily of bacterial origin [16]. HGT events are suspected to have played an important role in the emergence of plant parasitism in nematodes, enabling degradation of the plant cell wall, nutrient processing and manipulation of plant defences [17]. Due to their importance in the life cycle of plant-parasitic nematodes, a great deal of effort has been put into various approaches for effector identification, including genomic and transcriptomic analyses [10], transcriptomic analyses of purified gland cells [18] and proteomic analyses [19]. For some effectors, the likely biological functions, including host proteins targeted, have been identified [14, 20, 21].

Here, we report a high quality draft genome of a Ro1 isolate of G. rostochiensis, in combination with replicated transcriptome data from four key life stages, and genome sequence from eight populations across four pathotypes. We conducted whole genome comparisons between G. rostochiensis and related species [22-25] to explore the genomic and transcriptomic bases of pathogenicity. We discovered an unusually high frequency of 
well-supported non-canonical splice sites in G. rostochiensis, and found that this phenomenon was also present in related parasitic nematode species. Using an HGT analysis pipeline, we identified hundreds of genes in the G. rostochiensis genome that may have been acquired by gene transfers from non-metazoan origin, some of which likely play important roles in plant parasitism. We identified effectors in G. rostochiensis and found that they frequently grouped together into 'effector islands'. To explore the genetic bases of virulence, we compared genetic variation in effectors and other genes between pathotypes and found that effectors, in general, contained more non-synonymous mutations. Using the identified G. rostochiensis effectors as a training set, we identified a putative 'DOrsal Gland promoter element', or DOG box, which was also associated with effectors in related species. We were able to use the DOG box to predict novel effectors, confirmed by in situ hybridisation, in G. rostochiensis, and to identify all putative DOG effectors from available cyst nematode genomes.

\section{Results and discussion}

\section{The genome sequence of Globodera rostochiensis Ro1}

The genome of the potato cyst nematode, G. rostochiensis, pathotype Ro1 from the James Hutton Institute collection, was sequenced to 435.6-fold coverage and assembled into a high quality draft assembly (nGr.v1.0) of $95.9 \mathrm{Mb}$ (Table 1), consistent with experimental estimates of Globodera genome size [26]. The assembly shows a smaller size and total gene number, yet higher completeness than the G. pallida genome [22] (Table 1). Further, the low level of duplication of core, conserved genes (Table 1), and indeed of all genes (Additional file 1: Figure S1), suggest that the G. rostochiensis genome assembly is a more accurate representation of a Globodera genome, probably reflective of the low genetic variation present in the UK G. rostochiensis used for sequencing [11].

\section{Collaborative manual gene refinement reveals a uniquely} high frequency of non-canonical splice sites in Globodera To produce a high quality set of gene predictions, an initial phase of automated annotation was followed by manual refinement of approximately one-eighth of all gene models in the collaborative genome annotation editor WebApollo (Additional file 2: Supplementary information file 1). During the manual annotation phase, we noted that correction of many exon-intron boundaries to be consistent with mapped RNA-sequencing (RNA-seq) data (Fig. 1a) was only possible using noncanonical 5' donor splice sites (GC rather than GT). The frequency of GC-bearing introns in the manual annotation set was two orders of magnitude higher
Table 1 Genome statistics

\begin{tabular}{lll}
\hline & G. pallida & G. rostochiensis \\
\hline Assembly version & $n G p . v 1.0$ & $n G r . v 1.0$ \\
Assembly size (Mb) & 124.6 & 95.9 \\
Scaffolds (n) & 6873 & 4377 \\
Scaffold N50 (bp) & 121,687 & 88,495 \\
Longest scaffold (bp) & 600,076 & 688,384 \\
Contig N50 (bp) & 11,611 & 11,371 \\
Longest contig (bp) & 93,564 & 111,501 \\
Span of N's in assembly (bp) & $21,024,229$ & $4,445,051$ \\
GC (\%) & 36.7 & 38.1 \\
CEGMA (Complete/Partial \%) & $74.19 / 80.65$ & $93.55 / 95.56$ \\
Average CEG gene number & $1.23 / 1.29$ & $1.15 / 1.24$ \\
(Complete/Partial) & & \\
Gene density (per Mb) & 132.2 & 149.9 \\
Genes (n) & 16,466 & 14,378 \\
Proteins (n) & 16,417 & 14,309 \\
Proteins w/Start and Stop & $14,580(88.81 \%)$ & $13,083(91.43 \%)$ \\
codon (n) & & \\
Non-canonical splice sites (\%) & $3.56 \%(\mathrm{n}=4059)$ & $3.46 \%$ ( $\mathrm{n}=3835)$ \\
PfamA domains (cutoff 1e-5) (n) & 8853 & 8397 \\
Best BLAST hit to nematode & 8886 & 8603 \\
proteins (1e-10) (n) & & \\
\hline
\end{tabular}

than in the initial automated predictions. However, genome-wide re-prediction, using manually curated genes as a training set and allowing for the prediction of non-canonical GC/AG introns, increased the frequency of $\mathrm{GC} / \mathrm{AG}$ introns to that of the manually annotated set (Additional file 3: Table S1) and markedly improved upon automated predictions (see Additional file 2: Supplementary information file 1).

The frequency of GC/AG introns in G. rostochiensis was $3.46 \%$, the highest reported for any nematode. In addition to the GT or GC dinucleotide, 5' donor sites are characterised by a nine-base consensus sequence, CAGG[T|C]AAGT (where the initial CAG is in the preceding exon [27]). Although variations in the 5' donor site sequence were found, G. rostochiensis $\mathrm{GC} /$ AG introns conformed equally well, if not better, to this consensus as did GT/AG introns (Fig. 1a and b). We derived a revised $5^{\prime}$ donor consensus for the predicted introns for both GC and GT 5' sites and found both intron classes to use AAGG[T|C]AAGT (where the first AAG is in the preceding exon). We identified a similarly high frequency of GC/AG introns in G. pallida (3.53\%), and Rotylenchulus reniformis (2.36 \%) (PRJNA214681, Showmaker et al., unpublished), a sedentary endoparasite of multiple crop plants that is in a sister group to Globodera in the Tylenchoidea (Additional file 4: Figure S2). While GC/AG introns were apparently 


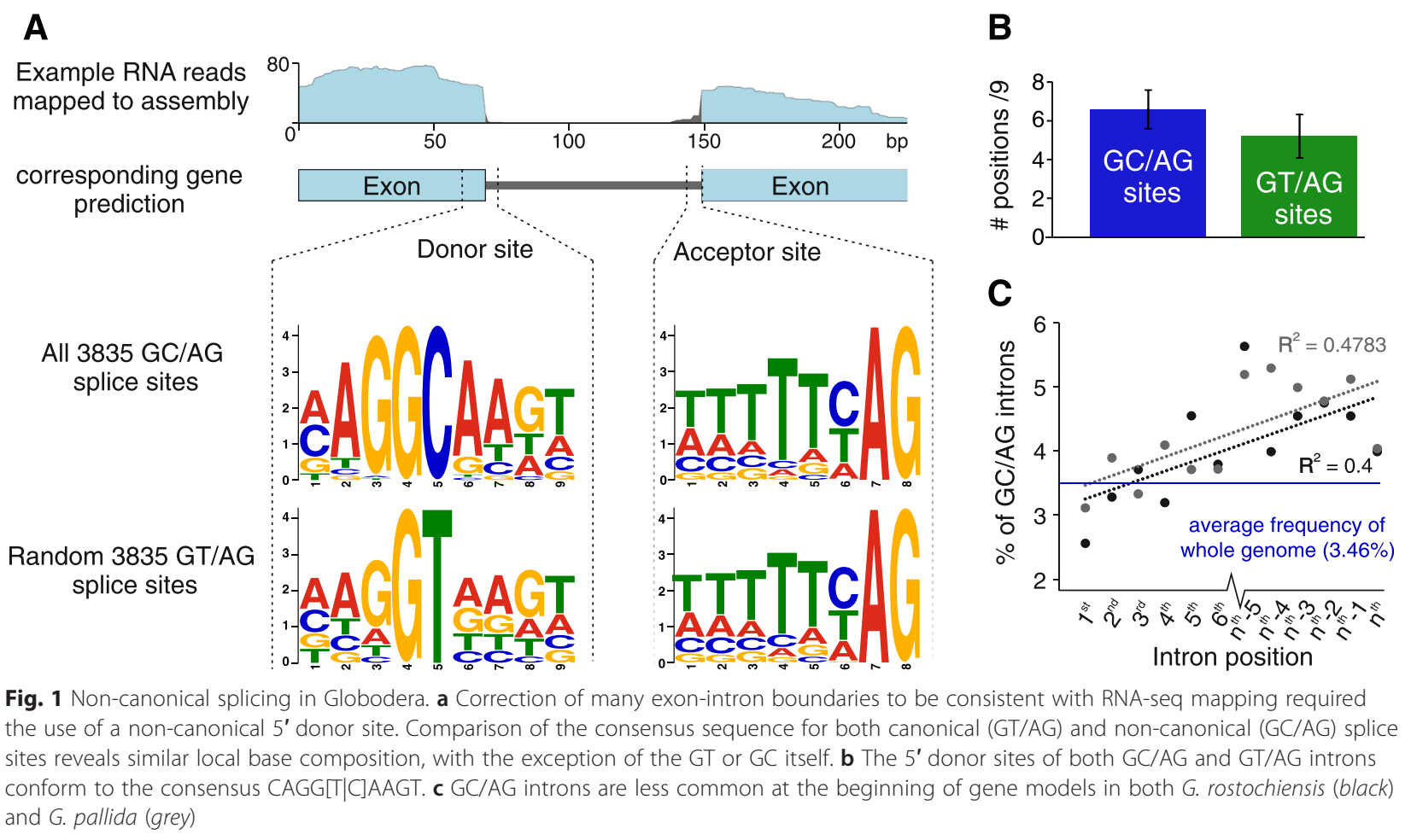

absent from the Meloidogyne species gene predictions, we suspect this may be due to restrictive settings during their annotation, as they are present in most species (Additional file 4: Figure S2). The elevated proportion of non-canonical GC/AG introns appear to be restricted to the Heteroderidae.

In species pairs with a low GC/AG intron frequency, such as Caenorhabditis elegans and the closely related C. briggsae, there is no obvious conservation of noncanonical splice site usage in their orthologous genes [28]. However, for genes in G. rostochiensis with at least one GC/AG intron, 72 \% of the corresponding one-toone orthologues in G. pallida also contained at least one $\mathrm{GC} / \mathrm{AG}$ intron $(\mathrm{n}=2148)$, compared to an average of $10.8 \%$ for identically sized subsets of non-GC/AG intron containing G. rostochiensis genes (1000 iterations, stdev $=0.8 \%)$. Within those genes, orthologous introns also tended to have conserved non-canonical splice sites. For $30 \%$ of the G. rostochiensis GC/AG introns in oneto-one orthologues, the corresponding G. pallida intron also used GC/AG. GC/AG introns had a biased distribution within genes in both species, tending to be less common in introns in the $5^{\prime}$ portion of genes compared to introns in the $3^{\prime}$ portion (Fig. 1c).

\section{Life stage specific transcriptome}

From the G. pallida genome project [22], it was clear that the key parasitic transitions to be captured in terms of all cyst nematode gene expression, and in particular for effectors, is from outside the plant (J2) compared to inside the plant (sedentary females). We used nematodes at 14 days post infection (dpi) as this provides an ideal intermediate for the sedentary stages: variation in gene expression at $14 \mathrm{dpi}$ accounts for most of the variation in gene expression at $7 \mathrm{dpi}(84 \%)$, and at $21 \mathrm{dpi}(60 \%$, Additional file 5: Figure S3). G. rostochiensis pathotype Ro1 gene expression was therefore analysed at four key stages across the life cycle: dormant cysts; hydrated eggs; hatched infective $\mathrm{J} 2$; and feeding parasitic females. Using a false discovery rate (FDR) of $<0.001$ and a minimum fold-change of 4, 6720 genes $(47 \%)$ were found to be differentially expressed. Differentially expressed genes were grouped into expression clusters; those that uniquely describe each life stage, two life stages or three life stages were identified (Fig. 2; expanded in Additional file 6: Figure S4; Additional file 7: File S1 contains the data matrix of normalised expression values). Some expression clusters showed a stepwise increase or decrease in expression corresponding to transitions through the life cycle. As much as $94 \%$ of all differentially expressed genes, and thus $\sim 44 \%$ of all genes, are manually grouped into 25 biologically relevant expression superclusters (Additional file 6: Figure S4).

G. rostochiensis predicted proteins were clustered with those from the cyst nematode G. pallida [20], the rootknot nematodes M. hapla [24] and M. incognita [23], the pine wilt nematode Bursaphelenchus xylophilus [29] and C. elegans (Fig. 3a; for relationships between these 


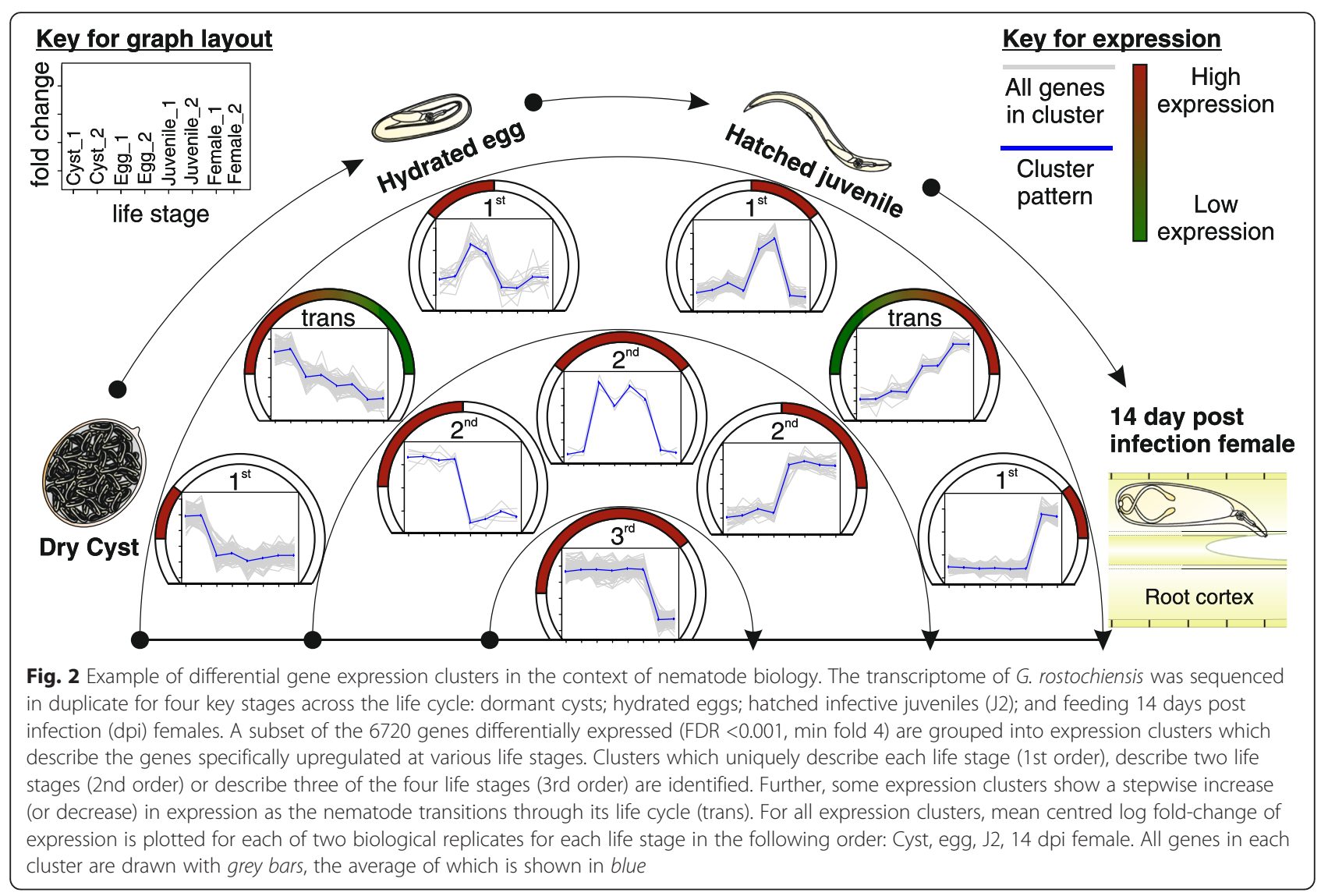

species see Additional file 4: Figure S2). Among the 16,821 OrthoMCL clusters, 2821 contained representatives from all nematodes tested, 220 clusters contained only proteins from plant parasites, 372 clusters contained only proteins of Globodera spp. and Meloidogyne spp. and 1986 clusters were composed solely of proteins from the cyst nematodes G. rostochiensis and G. pallida.

Focusing on these four categories of orthologous clusters (all nematodes, all plant parasites, Meloidogyne plus Globodera and Globodera) we correlated the orthologue definition and transcriptional clustering data to explore possible functional roles of genes unique to subsets of the taxa analysed. Only $34 \%$ of genes in clusters with members from all five nematodes, or clusters lacking only C. elegans, were differentially expressed, compared to $47 \%$ differentially expressed overall (Fig. 3b), congruent with the assumption that these families are likely to include loci with roles in core physiology. Interestingly however, genes specifically upregulated in eggs contain a higher relative abundance of genes in orthologous clusters common to all plant parasites yet absent in $C$. elegans, compared to other orthologous gene categories (Fig. 3c).

Only $43 \%$ of genes in orthologous clusters private to Meloidogyne and Globodera were differentially expressed. In contrast, of the genes in orthologous clusters only present in the two Globodera species, $60 \%$ were differentially regulated, suggesting that these genes play a dynamic role in parasite development. Furthermore, over two-fifths of genes (42\%) that are differentially regulated in the infective juvenile stage of $G$. rostochiensis are those that are unique to the Globodera. Expression super-clusters 13 and 24, which describe those genes specifically upregulated or downregulated in the infective juvenile stage, respectively, contain a higher relative abundance of genes in orthologous clusters unique to Globodera species compared to other orthologous gene categories (Fig. 3c).

G. rostochiensis proteins in clusters private to Meloidogyne and Globodera were enriched for GO terms associated with gene silencing by miRNA $(p<0.001$, FDR 0.05), including nine proteins with highest similarity to worm-specific argonautes (WAGOs) in C. elegans. WAGOs are central to the RNAi pathway, being responsible for binding of small RNAs and mediation interactions with other proteins, and show an exceptional diversity within the phylum Nematoda. It has been suggested that the expansion of WAGOs within Nematoda is associated with extreme functional plasticity [30]. Enrichment of WAGOs in the Meloidogyne and Globodera lineage, in combination with phylogenetically distinct clades of WAGOs in the Heteroderidae (Additional file 8: Figure S5), may indicate functional 


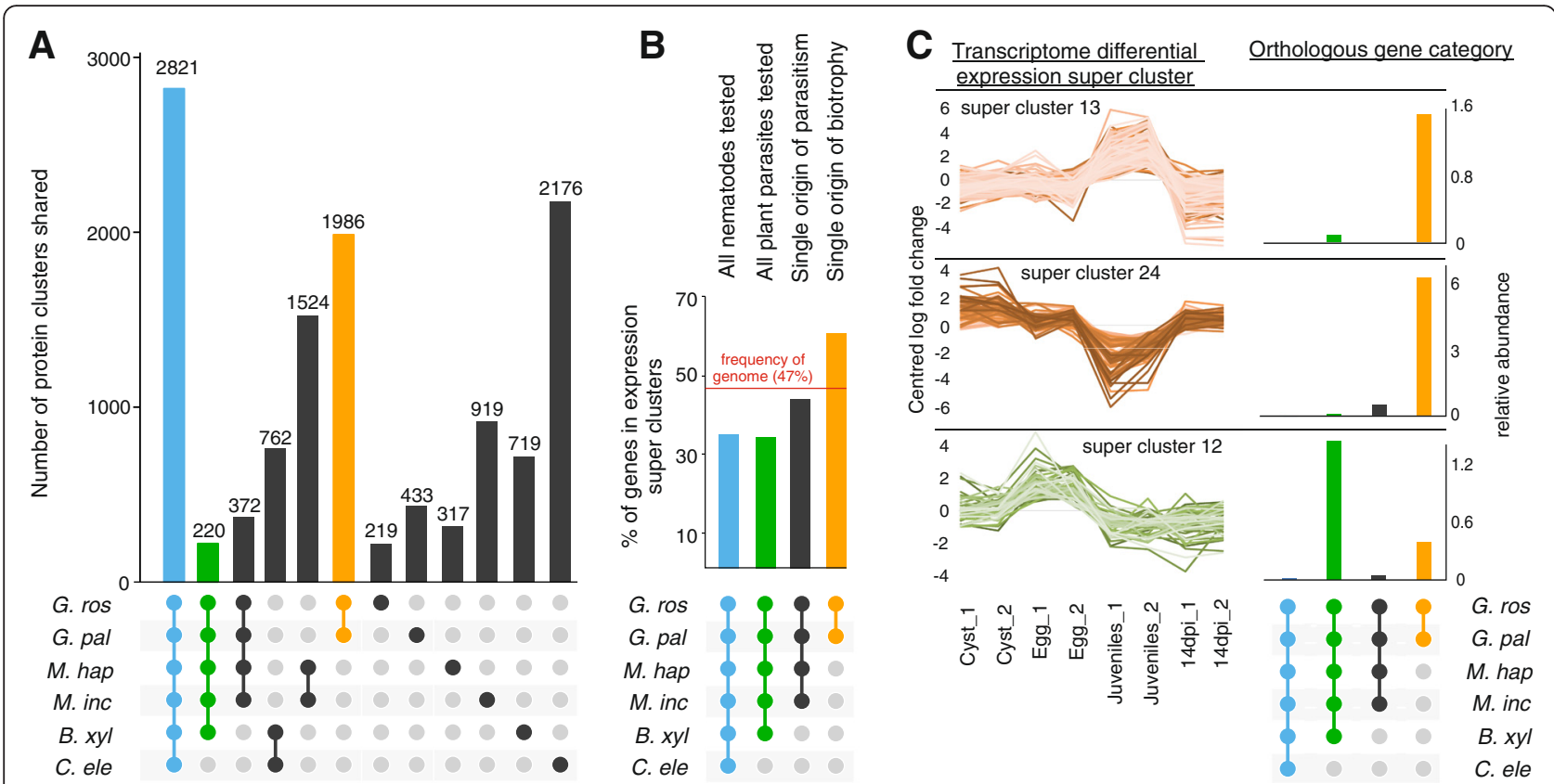

Fig. 3 Putative orthologous gene clusters in related nematode species and transcriptomic analysis. a The predicted protein sets of five plant parasites, from two independent origins of parasitism, were compared to that of the free living nematode $C$. elegans. The analysis includes two cyst nematodes, G. rostochiensis and G. pallida, two root knot nematodes, M. hapla and M. incognita, the pine wilt nematode B. xylophilus and the free living nematode $C$. elegans. The histogram shows the number of clusters shared uniquely between the species highlighted below. A total of 1986 clusters of genes are present in both cyst nematode species and absent from all other nematodes tested (orange). b Focusing on four orthologous gene cluster categories (all nematodes, all plant parasites, a single origin of plant parasitism and a single origin of sedentary biotrophy), the percentage of genes present in differential super-clusters suggest that those genes unique to the Globodera are preferentially differentially regulated throughout the adapted life cycle. c Analysing the relative abundance of genes from each orthologous gene cluster category in each of the differential expression super-clusters suggests that those genes specifically differentially regulated during the infective juvenile stage are those unique to the Globodera

diversification following expansion. With the exception of GROS_g08854, all G. rostochiensis WAGOs that are differentially regulated are present in differential expression super-clusters 19, 20 and 21. All but one of these differentially expressed WAGOs are in Clades $1 /$ 2/4/5 and 10/11. Expression super-clusters 19, 20 and 21 are characterised by significant upregulation at 14 dpi, suggesting a dynamic role for WAGO clade 1/2/4/ 5 and $10 / 11$ as $G$. rostochiensis transitions through parasitism.

\section{Genes acquired by horizontal transfer have substantially contributed to the genome of $G$. rostochiensis}

Horizontal gene transfer (HGT) events have played an important role in the emergence of plant-parasitism in nematodes [17]. Numerous plant cell wall degrading enzymes, originally acquired from bacteria, are present in a wide range of tylenchomorph plant-parasitic nematode species, while diplogasterid nematodes have acquired functionally analogous genes from fungi [17]. Using a systematic genome-wide approach, putative HGT events were identified based on the ratio of their sequence similarity to metazoan and non-metazoan sequences (Alien Index (AI), (Alienness [31-33])). Proteins with an $\mathrm{AI}>0$ and more than $70 \%$ identity to a non-metazoan sequence were considered putative contaminants $(n=18)$ and not included in these analyses.

We identified $519 \mathrm{G}$. rostochiensis proteins that may have originated through HGT events $(\mathrm{AI}>0)$, including all previously published cases of HGT into cyst nematodes present in the predicted proteins (Table 2). Of the 519 genes putatively acquired by HGT, $87 \%$ have some evidence of transcription at the four life stages sampled (cumulative FPKM $>1$, c.f. $95 \%$ of all proteins), $91 \%$ have at least one intron (c.f. $95 \%$ of all proteins) and $92 \%$ are on scaffolds containing other genes not predicted to be acquired by HGT (c.f. $95 \%$ of random set ( $\mathrm{n}=519), 1000$ iterations). We found strong support (AI $>30$ ) for 91 proteins (Additional file 9: Table S2). In $77 \%$ of these cases (70/91), the most similar sequences identified were of bacterial origin, while in $~ 11 \%$ (10/ 91), the most similar sequences were of fungal origin, consistent with previous reports of HGT in plantparasitic nematodes. The remaining proteins with an AI $>30$ had closest similarity to proteins from protists $(n=$ $7)$, plant $(n=3)$ and a virus $(n=1)$. No phylogenetically 
Table 2 Genes acquired via HGT in other cyst and root-knot nematodes also found in the genome of G. rostochiensis

\begin{tabular}{|c|c|c|c|c|c|c|}
\hline Process & Gene family & Function & Pfam domains & $\begin{array}{l}\text { Highest } \\
\text { Al }\end{array}$ & Reference & $\begin{array}{l}\text { G. } \\
\text { rostochiensis } \\
\text { genes }\end{array}$ \\
\hline \multirow{22}{*}{$\begin{array}{l}\text { Cell wall } \\
\text { degradation }\end{array}$} & \multirow[t]{11}{*}{ GH5_2 Cellulases } & \multirow[t]{11}{*}{ Cellulose degradation } & \multirow{11}{*}{$\begin{array}{l}\text { PF00150 } \\
\text { Cellulase } \\
\text { (glycosyl hydrolase family 5) }\end{array}$} & \multirow[t]{11}{*}{198.94} & \multirow[t]{11}{*}[12]{} & GROS_g01454 \\
\hline & & & & & & GROS_g04677 \\
\hline & & & & & & GROS_g05961 \\
\hline & & & & & & GROS_g05962 \\
\hline & & & & & & GROS_g07338 \\
\hline & & & & & & GROS_g07446 \\
\hline & & & & & & GROS_g07949 \\
\hline & & & & & & GROS_g10505 \\
\hline & & & & & & GROS_g11008 \\
\hline & & & & & & GROS_g11200 \\
\hline & & & & & & GROS_g11949 \\
\hline & \multirow[t]{7}{*}{ Expansin-like proteins } & \multirow{7}{*}{$\begin{array}{l}\text { Softening of non-covalent } \\
\text { bonds }\end{array}$} & \multirow{7}{*}{$\begin{array}{l}\text { PF03330 } \\
\text { Rare lipoprotein A (RlpA)-like } \\
\text { double-psi beta-barrel }\end{array}$} & \multirow[t]{7}{*}{29.93} & \multirow[t]{7}{*}{ [90] } & GROS_g03476 \\
\hline & & & & & & GROS_g09961 \\
\hline & & & & & & GROS_g10585 \\
\hline & & & & & & GROS_g11726 \\
\hline & & & & & & GROS_g11727 \\
\hline & & & & & & GROS_g12817 \\
\hline & & & & & & GROS_g12966 \\
\hline & $\begin{array}{l}\text { GH53 candidate Arabinogalactan } \\
\text { endo-1,4-beta-galactosidase }\end{array}$ & $\begin{array}{l}\text { Pectinose/arabinogalactan } \\
\text { degradation }\end{array}$ & $\begin{array}{l}\text { PF07745 } \\
\text { Glycosyl hydrolase family } 53\end{array}$ & 349.30 & [91] & GROS_g08150 \\
\hline & \multirow[t]{3}{*}{ PL3 Pecate lyase } & \multirow[t]{3}{*}{ Pectin degradation } & \multirow{3}{*}{$\begin{array}{l}\text { PF03211 } \\
\text { Pectate lyase }\end{array}$} & \multirow[t]{3}{*}{137.06} & \multirow[t]{3}{*}[92,93]{} & GROS_g04366 \\
\hline & & & & & & GROS_g05398 \\
\hline & & & & & & GROS_g07968 \\
\hline \multirow[t]{4}{*}{$\begin{array}{l}\text { Plant defense } \\
\text { manipulation }\end{array}$} & GH18 chitinase & Chitin degradation & $\begin{array}{l}\text { PF00704 } \\
\text { Glycosyl hydrolase family } 18\end{array}$ & 2.30 & [94] & GROS_g11136 \\
\hline & \multirow[t]{2}{*}{ Chorismate mutase } & \multirow{2}{*}{$\begin{array}{l}\text { Conversion of Chorismate } \\
\text { into SA }\end{array}$} & \multirow{2}{*}{$\begin{array}{l}\text { PF01817 } \\
\text { Chorismate mutase type ॥ }\end{array}$} & \multirow[t]{2}{*}{42.36} & \multirow[t]{2}{*}{ [95] } & GROS_g02441 \\
\hline & & & & & & GROS_g08190 \\
\hline & Candidate Isochorismatase & $\begin{array}{l}\text { Conversion of Chorismate } \\
\text { into SA }\end{array}$ & $\begin{array}{l}\text { PF00857 } \\
\text { Isochorismatase family }\end{array}$ & 66.08 & {$[96]$} & GROS_g01640 \\
\hline Detoxification & Candidate Cyanate lyase & & $\begin{array}{l}\text { PF02560 } \\
\text { Cyanate lyase C-terminal } \\
\text { domain }\end{array}$ & 11.51 & {$[17,24]$} & GROS_g09531 \\
\hline \multirow{11}{*}{$\begin{array}{l}\text { Nutrient } \\
\text { processing }\end{array}$} & \multirow[t]{11}{*}{ GH32 invertase } & \multirow{11}{*}{$\begin{array}{l}\text { Degradation of sucrose in } \\
\text { glucose and fructose }\end{array}$} & \multirow{11}{*}{$\begin{array}{l}\text { PF00251 } \\
\text { Glycosyl hydrolases family } \\
32 \text { N-terminal domain }\end{array}$} & 241.26 & {$[22,23]$} & GROS_g05724 \\
\hline & & & & & & GROS_g06434 \\
\hline & & & & & & GROS_g08674 \\
\hline & & & & & & GROS_g09735 \\
\hline & & & & & & GROS_g09969 \\
\hline & & & & & & GROS_g10583 \\
\hline & & & & & & GROS_g11374 \\
\hline & & & & & & GROS_g11397 \\
\hline & & & & & & GROS_g11793 \\
\hline & & & & & & GROS_g13274 \\
\hline & & & & & & GROS_g14232 \\
\hline
\end{tabular}


Table 2 Genes acquired via HGT in other cyst and root-knot nematodes also found in the genome of G. rostochiensis (Continued)

\begin{tabular}{|c|c|c|c|c|c|c|}
\hline & VB1 thiD & Vitamin B1 biosynthesis & $\begin{array}{l}\text { PF08543 } \\
\text { Phosphomethylpyrimidine } \\
\text { kinase }\end{array}$ & 154.50 & [97] & GROS_g07352 \\
\hline & VB1 thiE & Vitamin B1 biosynthesis & $\begin{array}{l}\text { PF02581 } \\
\text { Thiamine monophosphate } \\
\text { synthase/TENI }\end{array}$ & 163.99 & [97] & GROS_g07353 \\
\hline & VB1 thi4 & Vitamin B1 biosynthesis & $\begin{array}{l}\text { PF01946 } \\
\text { Thi4 family }\end{array}$ & 108.07 & [97] & GROS_g10855 \\
\hline & VB1 thiM & Vitamin B1 salvage & PF02110 & 46.05 & [97] & GROS_g07354 \\
\hline & & & $\begin{array}{l}\text { Hydroxyethylthiazole kinase } \\
\text { family }\end{array}$ & & & \\
\hline & VB1 tenA & Vitamin B1 salvage & PF03070 & 108.33 & [97] & GROS_g05327 \\
\hline & & & TENA/THI-4/PQQC family & & & GROS_g07355 \\
\hline & VB5 panC & Vitamin B5 biosynthesis & PF02569 & 183.11 & [97] & GROS_g05752 \\
\hline & & & Pantoate-beta-alanine ligase & & & \\
\hline & VB6 aSNO & Vitamin B6 biosynthesis & PF01680 & 12.72 & [98] & GROS_g08956 \\
\hline & & & SOR/SNZ family & & & \\
\hline & Candidate PolS Polyglutamate & Not known & PF09587 & 102.00 & [99] & GROS_g07961 \\
\hline & & & $\begin{array}{l}\text { Bacterial capsule synthesis } \\
\text { protein PGA_cap }\end{array}$ & & & \\
\hline & Candidate GSI Glutamine synthase & Nitrogen assimilation & PF00120 & 29.24 & {$[100,101]$} & GROS_g02362 \\
\hline & & & $\begin{array}{l}\text { Glutamine synthetase, } \\
\text { catalytic domain }\end{array}$ & & & \\
\hline $\begin{array}{l}\text { Feeding site } \\
\text { induction }\end{array}$ & NodL - like & $\begin{array}{l}\text { Candidate } \\
\text { acetyltransferase }\end{array}$ & $\begin{array}{l}\text { PF12464 } \\
\text { Maltose acetyltransferase }\end{array}$ & 13.12 & {$[100,102]$} & GROS_g11033 \\
\hline & & & $\begin{array}{l}\text { PF00132 } \\
\text { Bacterial transferase } \\
\text { hexapeptide (six repeats) }\end{array}$ & & & \\
\hline Not known & Candidate L-threonine aldolase & $? ?$ & PF01212 & 164.69 & [100] & GROS_g10421 \\
\hline & & & Beta-elımınatıng lyase & & & GROS_g10422 \\
\hline & & & & & & GROS_g10423 \\
\hline & Candidate Phosphorybosyl & $? ?$ & PF00156 & 198.13 & {$[100,101]$} & GROS_g04632 \\
\hline & transterase & & $\begin{array}{l}\text { Phosphoribosyl transterase } \\
\text { domain }\end{array}$ & & & GROS_g06735 \\
\hline
\end{tabular}

confirmed HGT of protist, plant or virus origin has been identified to date in plant-parasitic nematodes. Given that some of these candidates are among genes with evidence of expression, they deserve further investigation.

Protein domains were identified in $65 \%$ of the putative HGT proteins with an AI $>0$ and $88 \%$ of those with an AI $>30$ (Additional file 10: Table S3). The HGT candidates included a set, with AI >29, with predicted functions in plant cell wall modification and degradation, including GH5 cellulases, expansin-like proteins, GH53 candidate arabinogalactan endo-1,4beta-galactosidase and PL3 pectate lyases. Other cases of HGT may be involved in nutrient processing. A GH32 protein from G. pallida has been shown to be a functional invertase expressed in the digestive system [34]. This enzyme may convert sucrose, the main circulating form of sugar in plants, into glucose and fructose which are readily usable by nematodes. We identified 11 GH32-bearing proteins in G. rostochiensis, suggesting that this function may be especially important. The phylogenetically dynamic pattern of HGT into tylenchomorph genomes is illustrated by the absence of GH30_8 xylanases, GH28 polygalacturonase as well as GH43 candidate arabinanase in G. rostochiensis and G. pallida, despite their presence in root-knot nematodes [16]. Furthermore, of the 91 genes with $\mathrm{AI}>30$, six are present in orthologous protein clusters unique to the Globodera and Meloidogyne, yet many classes are functionally represented in both genera, consistent with multiple acquisitions. The distribution of putative HGT cases across orthologous gene categories is broadly consistent between $\mathrm{AI}>0$ and $\mathrm{AI}>30$. Both suggest a substantial proportion of genes putatively acquired by HGT (36-45 \%) are unique to the Globodera and may 
give an insight into the relatively recent HGT history since the Globodera-Meloidogyne divergence (Fig. 4a). Threequarters of genes with $\mathrm{AI}>30$ and unique to Globodera are present in differential expression super-clusters, the most common of which are super-clusters 13 and 20 which, respectively, describe genes specifically upregulated during infective $\mathrm{J} 2$ and parasitic females. This may indicate that these genes (several candidate invertases, candidate L-threonine aldolase and VB1 tenA (Additional file 10: Table S3)) are functionally deployed during parasitism following horizontal transfer. Although transposable elements (TEs) are closely associated with putative HGT events $(p<0.001$, MannWhitney U test, Fig. $4 \mathrm{~b}$ ), the divergent transposable element assemblage in Globodera species compared to other tylenchomorphs (LINE/Jockey and SINE/Alu, Additional file 11: Figure S6) is not preferentially associated with putative HGT cases also specific to the Globodera (Fig. 4c).

\section{Effectors in G. rostochiensis are sequence diverse between pathotypes}

Effectors play central roles in both pathogenicity and virulence. The evolution of virulence on a particular host or variety can involve both gain and loss of effector function. Effectors may become specialised to function in a new host [35], while effector gene loss (or loss of expression) may allow a pathogen to evade recognition [36]. We identified G. rostochiensis effectors by sequence similarity to effectors with experimentally verified gland cell expression in related taxa (Heterodera, Globodera). Many effectors in plant-parasitic nematodes are members of large multi-gene families, only a subset of which are effectors [10, 13, 37]. For example, in G. pallida there are $\sim 300$ SPRY (PF00622) domain containing proteins, fewer than $10 \%$ of which are deployed as effectors [13]. We therefore further filtered the potential effector set for the presence of a signal peptide for secretion and absence of a transmembrane domain to retain a high confidence list of 138 loci (Additional file 12: Table S4), including 101 genes similar to sequences expressed in the dorsal gland cell, 35 genes similar to those expressed in subventral gland cells and two genes similar to those expressed in the amphid sheath cells. The set included representatives of 37 different effector gene families (Additional file 12: Table S4). The vast majority of these effectors (116/138) exhibited expression profiles consistent with a role in parasitism (Additional file 12: Table S4), as would be expected for effectors. The temporal expression profiles of dorsal and subventral effectors were also consistent with the observed changes in activity of these glands throughout nematode development [38-41]. Most subventral gland effectors were primarily expressed at $\mathrm{J} 2$, while dorsal gland effectors were expressed at $\mathrm{J} 2$ and/or 14 dpi. Approximately $8.5 \%$ of genes putatively acquired via HGT $(8.47 \%$ of
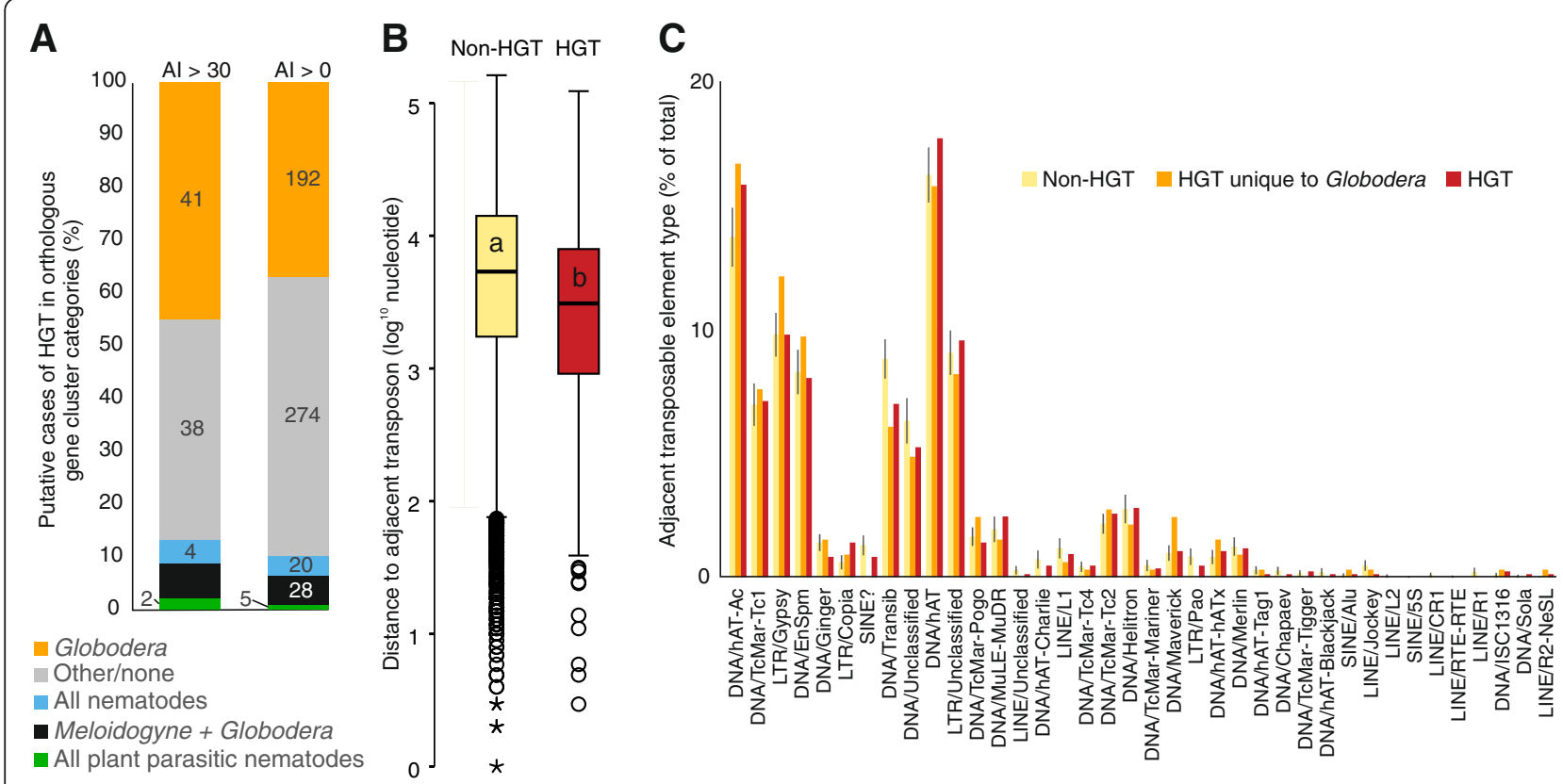

Fig. 4 Analysis of genes putatively acquired by HGT. a Using an Al $>30$ or $>0$, between $45 \%$ and $36 \%$ of putative HGT genes are present in orthologous gene cluster categories unique to the Globodera and may give an insight into the relatively recent HGT history since the Globodera-Meloidogyne divergence. $\mathbf{b}$ Genes putatively acquired by HGT (Al $>0$ ) are significantly closer to transposable elements when compared to all other genes not predicted to be acquired by HGT ( $p<0.000$, Mann-Whitney $U$ test). $\mathbf{c}$ There was no significant association of any independent class of transposable element with genes putatively acquired by HGT. Despite the divergent transposable element composition of Globodera (Additional file 11: Figure S6), these were not associated with putative HGT events specific to Globodera 
those with AI $>0$ and $8.79 \%$ of those with $>30$ ) are present on the stringent effector list; examples of which include putative pectate lyase, beta - 1,4 - endoglucanase and expansins.

Intra-species variation within the G. rostochiensis effectorome was examined by mapping whole genome resequencing data from nine populations across five pathotypes (Ro1, Ro2, Ro3, Ro4 and Ro5) to the reference assembly (pathotype Ro1). A total of 1,081,802 variants were detected, of which 794,505 were single nucleotide polymorphisms (SNPs) and 283,434 were insertions/deletions (indels) (Additional file 13: Table S5). Homozygous molecular markers descriptive of pathotypes 4 and 5 were identified (Additional file 14: Table S6). Interestingly, no variants were descriptive of all Ro1, Ro2 or Ro3 populations. Consistent with this, a maximum likelihood phylogeny constructed from 730,705 genome wide SNPs identifies two distinct groups of Ro1, together separate from Ro2, Ro3, Ro4 and Ro5 (Additional file 15: Figure S7A). The variation within pathotype Ro1 is as great as, if not greater than, the variation between Ro1 and the other pathotypes (Additional file 15: Figure S7B).

A total of $108 \mathrm{G}$. rostochiensis effectors (78\%) contained predicted modification of function (non-synonymous mutation) and/or predicted loss of function (frame shift indel or premature stop codon) in at least one pathotype. When accounting for gene length, $G$. rostochiensis effectors did not show significantly different numbers of predicted loss of function variants, but did contain significantly more total variants and more predicted modification of function variants per gene ( $n=131$, Mann-Whitney $\mathrm{U}$ test, $p<0.028$ and $p=$ 0.003 , respectively), compared to randomly selected non-effector genes. No individual variant was homozygous for the reference allele in all populations avirulent on H1 (Ro1 and Ro4) and homozygous for the variant allele in all populations virulent on H1 (Ro2, Ro3 and Ro5). This observation is consistent with the suggestion that distinct populations of Ro1 (Additional file 15: Figure S7 and [42]), in addition to Ro4, have evolved the same phenotype on $\mathrm{H} 1$ independently [8]. Convergent evolution of the same phenotype by independent mutations may be explained by identifying genes which are always homozygous present for at least one predicted loss or change of function variant in populations virulent on $\mathrm{H} 1$ and always homozygous absent for any predicted loss or change of function variants in populations avirulent on $\mathrm{H} 1$. No such cases were identified from these population sequencing data. However, 190 genes were identified with at least one predicted modification or loss of function variant homozygous absent in all avirulent populations and homozygous or heterozygous present in virulent populations. When cross-referenced with the high-confidence effector list, this was reduced to two genes. Gene g13394 is similar to
GLAND10 [43], which encodes a putative cellulose binding protein and originates from the subventral gland cell. Gene g12477 is similar to the 3H07_Ubiquitin_extension effectors that are expressed in the dorsal gland cell [44, 45], and are involved in host immune suppression [46]. Forty-eight SNPs were identified in 19 non-effector genes with a difference in average allele frequencies of $70 \%$ or higher between virulent and avirulent populations and a minimal difference in allele frequencies of $25 \%$ between individual virulent and avirulent populations (Additional file 16: Table S7), of which four SNPs were located in g03129, a Ryanodine receptor-like containing three SPRY domains, and seven in g09064, a molecular chaperone from the Hsp90 family.

\section{Effectors in the G. rostochiensis genome are compartmentalised into islands}

In several unrelated eukaryotic plant pathogens, effectors are not randomly distributed in the genome, but are rather located in specialised regions. For example, in Phytophthora infestans most effectors are located in gene-sparse regions of the genome and it is proposed that this facilitates rapid evolution and adaptation [47]. Comparatively, G. rostochiensis effectors were located in gene-dense regions of the genome (Fig. 5a), albeit with a skewed distribution of gene density compared to an identically sized subset of non-effectors (Student's $t$ test, $n=138, p<0.001$, Additional file 17: Figure S8). Compared to an expectation of $2 \%$ for a random set of 138 genes, the 138 high-confidence effectors had another high-confidence effector as an immediate chromosome neighbour in $22 \%$ of cases $\left(\chi^{2}, p<0.0005\right)$. This excess was due to local tandem duplication, as effectors that were directly adjacent to one another in the genome were often from the same effector family, and were frequently more similar to the adjacent gene than to other members of the same gene family located elsewhere in the genome. Such local tandem duplication is a common feature of gene families in G. rostochiensis (Fig. 5), however, groups of functionally related gene families (i.e. effectors) tend to be in clustered in genomic islands. For a random subset of 37 non-effector containing gene families, increasing distance from each gene reduces the likelihood of identifying another member in any of the same 37 families. However, the clustering of effector loci extends beyond immediate neighbours, with an excess of effector loci as next-buttwo neighbours $(\mathrm{n} \pm 3)$ and also at $\mathrm{n} \pm 6\left(\chi^{2}, p<0.01\right.$ and 0.001 , respectively, Fig. 5b). Over one-third of all effectors were described by 21 effector islands of 2-4 effector loci (Additional file 18: Table S8) with an average length of $\sim 20 \mathrm{~kb}$. Over half of the islands included effectors from more than one effector gene family, yet $80 \%$ comprised genes expressed in only one cell type (dorsal gland cell, subventral gland cell). Several islands 


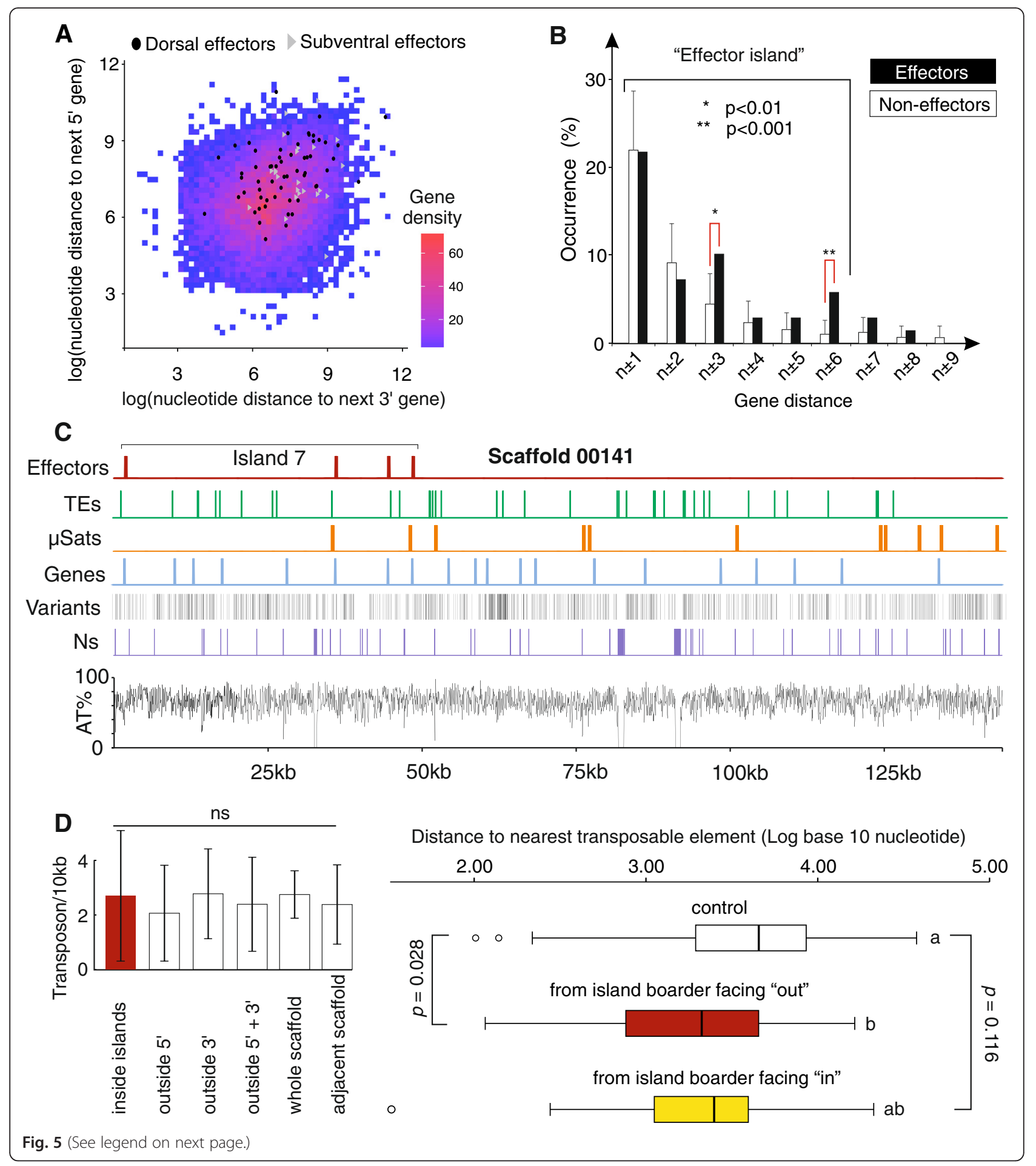




\section{(See figure on previous page.)}

Fig. 5 Effectors in G. rostochiensis are grouped into 'islands'. a Dorsal (black) or subventral (grey) effectors are skewed towards a higher neighbouring gene distance compared to random (Student's t-test, $p<0.01$ ), yet are contained within gene dense regions of the genome. b The presence of effectors in adjacent ( $n \pm 1$ ), or neighbouring positions (up to \pm 9 ), was determined. As a negative control, a subset of $612 \mathrm{G}$. rostochiensis gene families not predicted to contain effectors was identified from the OrthoMCL. Starting from this initial negative set of 612 gene families, 37 of these gene families were selected at random and the presence of genes from these 37 families in adjacent ( $n \pm 1$ ), or neighbouring positions (up to \pm 9 ), was determined. This process was repeated for 1000 iterations to generate a robust negative for the average frequency in each neighbouring position. The observed frequency of effector occurrence at each position (black bars) was compared to the average of 1000 iterations for the negative (white bars). For non-effector containing gene families, increasing distance from each gene reduces the likelihood of identifying another member in any of the same families (error bars indicate standard deviation of 1000 iterations). The clustering of effector loci extends beyond immediate neighbours, with an excess of effector loci as next-but-two neighbours $(n \pm 3)$ and also at $n \pm 6\left(x^{2}, p 0.01\right.$ and 0.001, respectively). $\mathbf{c}$ Example of one island (7) at the edge of scaffold 00141. With the exception of high effector density (red), no obvious genetic features are associated (gaps (Ns, purple), AT content (black line), gene density (blue) microsatellites (orange), variants (black bars) and transposable elements (TEs, green)). $\mathbf{d}$ No difference in transposon density was found between islands, in the remainder of scaffolds containing islands, in entire scaffolds containing islands or in scaffolds numerically adjacent to those containing islands (Kruskal-Wallis, $p=0.515$, error bards indicate standard deviation). When each island is treated as a single locus, the nearest external transposable element $5^{\prime}$ of the first gene, and $3^{\prime}$ of the last, is significantly closer than expected (ANOVA, $n=39, p=0.028$ accounting for multiple testing, Fig. $5 \mathrm{~d}$ ). Interestingly, the inverse measurement (the closest internal transposon to each island border), is not significantly closer than expected ( $n=45, p=0.116$, Fig. $5 \mathrm{~d}$ ), suggesting that this may be a feature of islands as an integral whole, rather than the separate genes comprising the islands

included loci similar to other effectors not included in the high-confidence list.

G. rostochiensis effector islands were also identified in G. pallida. Effector islands containing more than one one-to-one orthologue were similarly arranged in close proximity in G. rostochiensis and G. pallida, with just three exceptions. One island in G. rostochiensis was split across the ends of two scaffolds in G. pallida, suggesting the split in G. pallida may be an artefact of gapped assembly. Two other G. rostochiensis islands were dispersed in G. pallida, across different large scaffolds. Synteny between the genome assemblies of G. rostochiensis and G. pallida extends beyond effector islands, despite the fragmented nature of both assemblies. Based on OrthoMCL protein cluster-membership, 109 distinct syntenic clusters of scaffolds which contained runs of at least five syntenic proteins each were identified, involving $249 \mathrm{G}$. pallida and $202 \mathrm{G}$. rostochiensis scaffolds (Additional file 19: Figure S9). In total, $38.2 \mathrm{Mb}$ of G. pallida (36.9 \% of the genome) scaffolds are partially syntenic to $31.1 \mathrm{Mb}(34.0 \%$ of the genome) of G. rostochiensis scaffolds (ignoring N's). Breakage of synteny between two scaffolds was observed in 20 pairs, seven of which involved inversions. The low proportion of syntenic regions most likely reflects the draft nature of both assemblies (G. pallida scaffolds in clusters: $12 \% \mathrm{~N}$ 's; G. rostochiensis scaffolds in clusters: $4.9 \%$ N's). A subset of the largest syntenic cluster is shown in Fig. 6. Synteny breakpoints which primarily co-occur with large insertions in the G. pallida assembly may suggest that large-scale rearrangements have taken place during their divergence and yet effector islands remain predominantly intact. Long-range DNAsequencing data will prove crucial for assessing the true proportion of syntenic scaffolds and estimating the amount of synteny breakage.
Identifying features enriched within effector islands in G. rostochiensis remains challenging; there is no evidence for more AT-rich sequences, contig break points, polymorphisms or microsatellite repeats within islands, flanking islands or scaffolds containing islands (Fig. 5c). However, despite no difference in transposon density within islands $(2.7 / 10 \mathrm{~kb} \pm 2.4)$, in the remainder of scaffolds containing islands $(2.4 / 10 \mathrm{~kb} \pm 1.7)$, in entire scaffolds containing islands $(2.8 / 10 \mathrm{~kb} \pm 0.9)$ or in scaffolds numerically adjacent to those containing islands

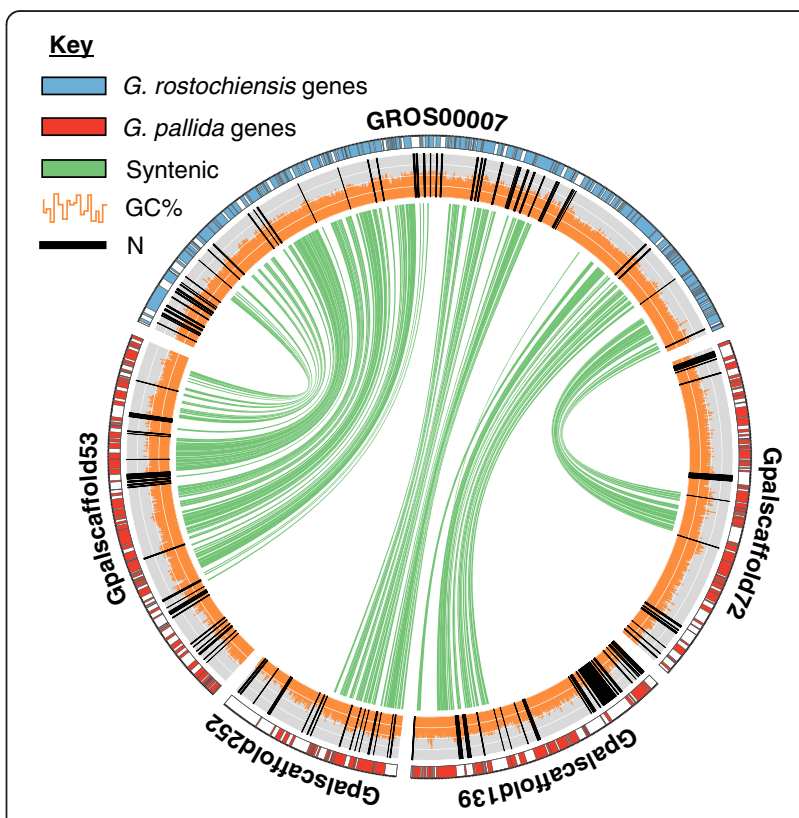

Fig. 6 Synteny between G. rostochiensis and G. pallida. G. rostochiensis genes (blue) in scaffold7 (500 kb) are syntenic (green arcs) with G. pallida genes (red) on four scaffolds. Synteny breakpoints primarily co-occur with large insertions in the G. pallida assembly. GC content and regions of undetermined sequence are represented by orange and black bars, respectively 
(2.4/10 kb \pm 1.5 , Kruskal-Wallis, $p=0.515$, Fig. 5d), transposable elements are closely associated to island borders. When each island is treated as a single locus, the nearest external transposable element $5^{\prime}$ of the first gene, and $3^{\prime}$ of the last, is significantly closer than expected (ANOVA, $n=39, p=0.028$ accounting for multiple testing, Fig. 5d). Interestingly, the inverse measurement (the closest internal transposon to each island border), is not significantly closer than expected $(\mathrm{n}=45, p=0.116$, Fig. 5d), suggesting that this may be a feature of islands as an integral whole, rather than the separate genes comprising the islands.

\section{Identification of a putative enhancer motif associated with dorsal gland effectors}

The existing roster of effector proteins in plant-parasitic nematodes has been defined through painstaking and exacting experimental studies employing gland cellspecific complementary DNA (cDNA) sequencing and in situ hybridisation [43]. We therefore sought possible regulatory motifs associated with the highly tissue specific expression pattern of effector genes that might act as an alternative criterion for their identification in silico $[10,48]$. By employing a differential motif discovery algorithm which normalises for GC content (HOMER)
[49], we identified a short DNA motif (the DOrsal Gland motif or DOG box, ATGCCA), specifically enriched in the promoter region (500 bp upstream of the start codon) of genes sequence-similar to experimentally validated dorsal gland cell effectors, compared to either sub-ventral gland effectors or all other non-effectors $\left(p=1 \mathrm{e}^{-10}\right)$. Of the $101 \mathrm{G}$. rostochiensis dorsal gland effectors, $77 \%$ had at least one DOG box in their promoter region. This encompasses 26 of the 28 dorsal gland effector families (92\%) including genes that are unrelated in sequence and ontogeny, yet only 5/10 non-dorsal gland effectors (subventral and amphid). Dorsal gland effectors contained an average of 2.54 DOG boxes in their promoter regions, compared to 0.22 for an identically sized subset of non-effectors, 0.32 for all non-effectors or 0.48 for effectors secreted from subventral glands (Fig. 7a). Motif occurrence peaked $150 \mathrm{bp}$ upstream of the start codon and was not strand-specific. Despite the presence of an ATG within the DOG box, the motif does not arise from specifically mis-predicting the start codon of effectors. A strand-specific, Kozak-like motif peak which includes the start codon (AAAATG) was observed in dorsal, subventral and non-effectors at the predicted start of the coding sequence (Fig. 7b). We were unable to identify a motif that correlated with

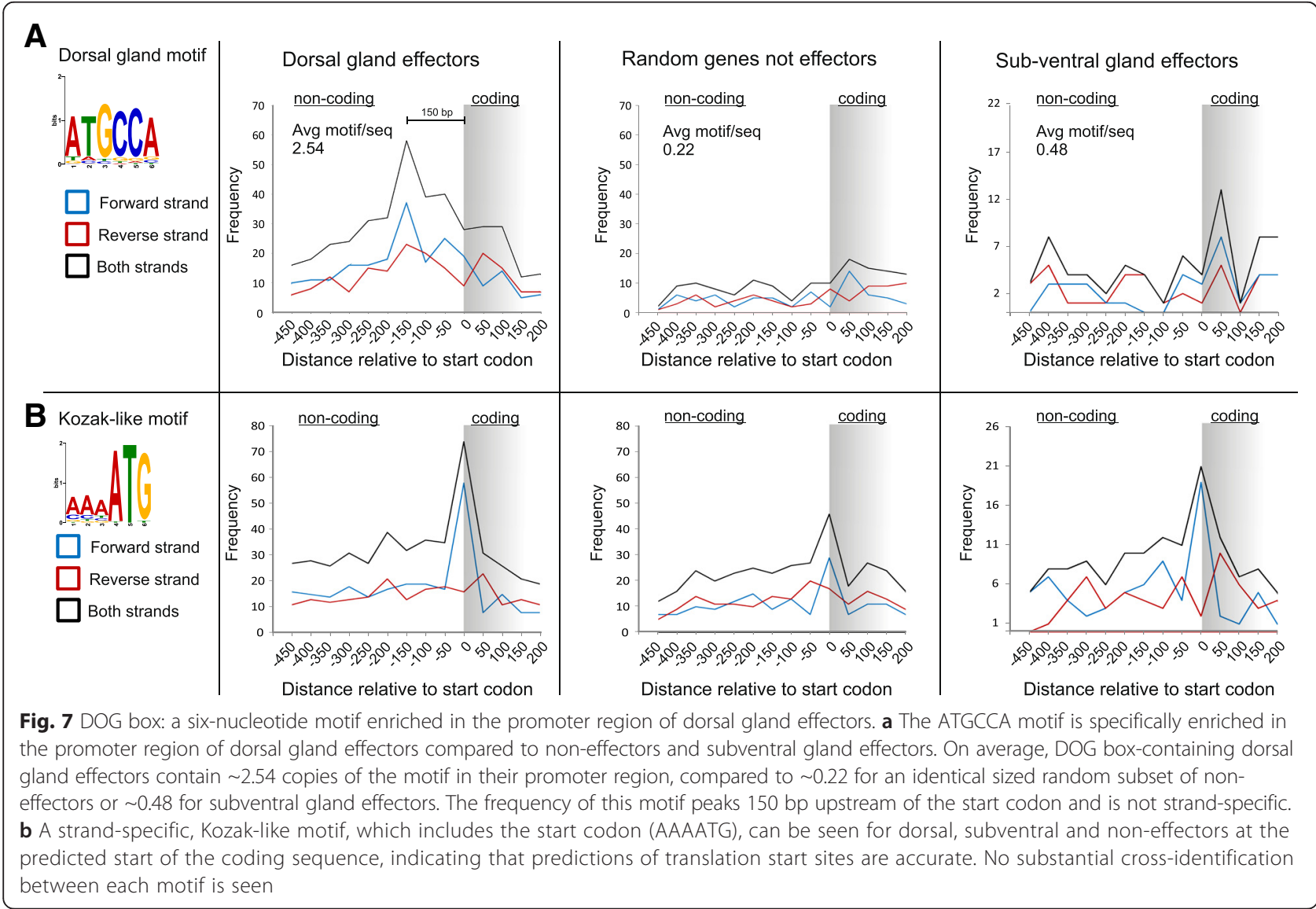


time of expression (e.g. when comparing dorsal gland effectors expressed at 14 dpi versus dorsal gland effectors expressed at J2) or with expression in the subventral gland. We found no enrichment of DOG boxes upstream of the first gene in tandem series of adjacent dorsal gland cell-expressed effectors arranged in an island.

\section{The DOG box as a predictor of effectors}

We screened the regions 500 bp upstream of all loci in the G. rostochiensis genome for DOG boxes on either strand. The number of genes associated with multiple DOG boxes was significantly higher than expected by chance (Fig. 8a). For some genes, nearly one-fifth of the entire 500 bp promoter region comprised ATGCCA motifs. Sequences with more DOG boxes in their promoter regions were more likely to have predicted a signal peptide for secretion (Fig. 8b). These findings suggest that the DOG box may be a strong predictor of secretion, and thus likely effector function, of $G$. rostochiensis genes. The same DOG box motif was also present at a significantly higher frequency than would be expected by chance and was preferentially associated with secreted proteins in G. pallida (Fig. 8a, b). In the more distantly related $M$. hapla, the number of genes with multiple occurrences of the motif in their promoter region is higher than expected by chance, but the presence of motifs was not associated with the downstream gene encoding a predicted signal peptide. No enrichment of the DOG box or association with secreted proteins was observed for the much more distantly related B. xylophilus. This suggests that in addition to minimal overlap between effector repertoires [22, 50], the control of effector expression in the dorsal gland cell may also require a different motif/s in these nematodes.

Although not all secreted proteins are effectors, all effector proteins are secreted. Within the $150 \mathrm{G}$. rostochiensis genes with three or more DOG boxes and a signal peptide, there were 31 known effectors from 14 families, an approximately 100 -fold enrichment. The expression patterns of these 150 genes (including newly discovered candidate effector sequences) were consistent with a role in parasitism. For G. pallida, where more comprehensive life stage expression data are available, the same association was observed (Additional file 20: Figure S10) [22]. Despite the fact that most genes with $>3$ ATGCCA motifs in G. pallida and a signal peptide are expressed at $\mathrm{J} 2$, the number of motifs in the promoter region was not a quantitative predictor of gene expression at J2 $\left(R^{2}=0.0002\right.$, Additional file 20: Figure S10) or at any other life stage, indicating that the ATGCCA motif is not a J2 enhancer. These data most likely reflect the biology of the nematode which dictates that a substantial proportion of effectors are required in the dorsal gland during the infective juvenile stages.

We used an extended set of criteria to predict potential DOG effectors from G. rostochiensis and G. pallida. Genes with two or more DOG box motifs within $500 \mathrm{bp}$ upstream of the start codon, a signal peptide and no transmembrane domain on the corresponding protein,

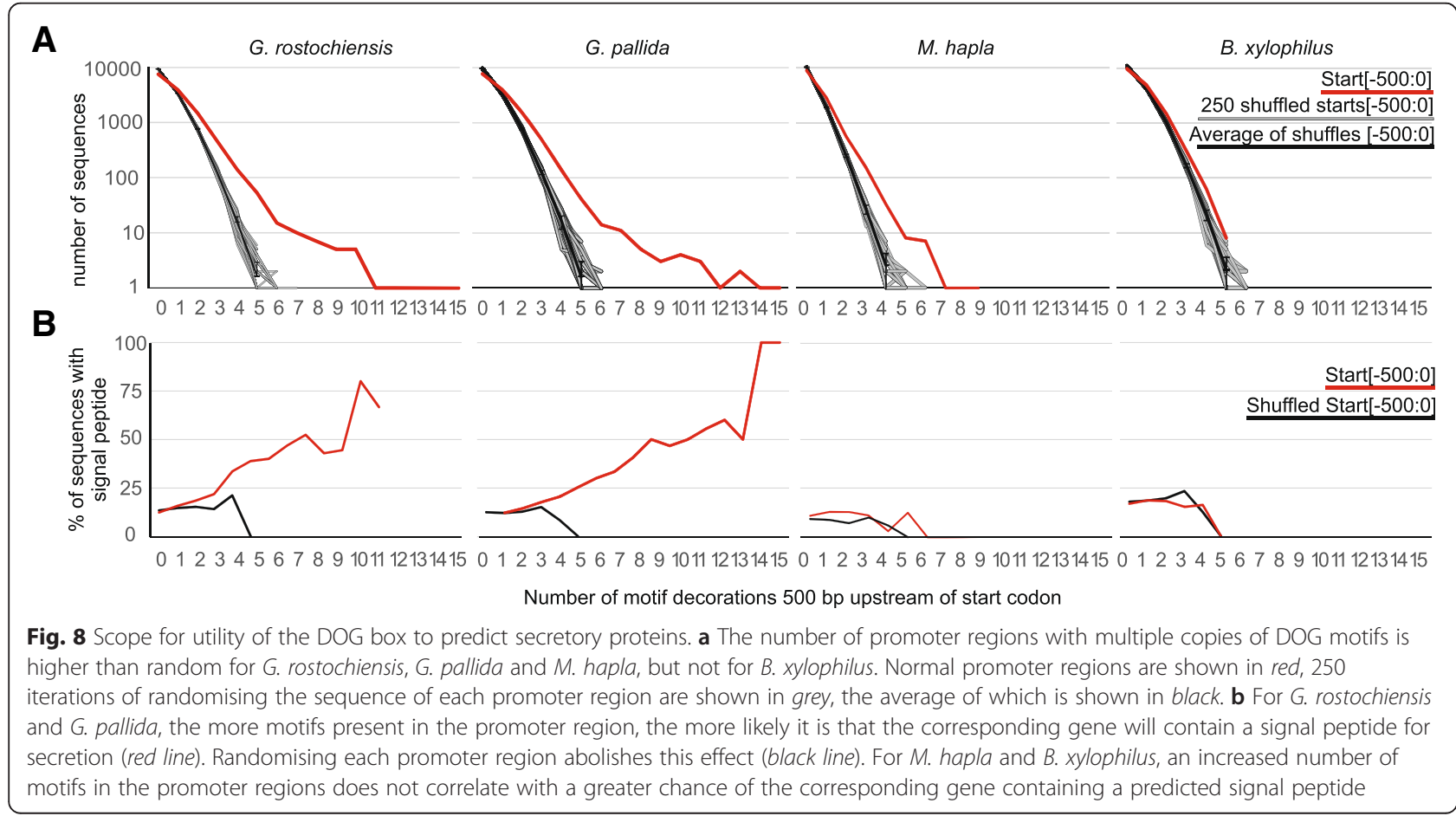


and temporal expression profiles consistent with a role in parasitism (Fig. 9a, b, Additional file 21: Table S9 and Additional file 22: Table S10) were classified as likely effectors. To validate these criteria, we examined the spatial expression pattern using in situ hybridisation of two new predictions that had no similarity to any published effector. Both exhibited expression in the dorsal gland cell (Fig. 9c), confirming that the DOG box, in combination with other criteria, can act as a predictor of novel effector candidates. Novel gland cell protein g14226 was clustered in a genomic island with several other similar genes with multiple DOG boxes in their promoter region, another signature of canonical Globodera effectors. As biological understanding of dorsal (and other) gland expression in tylenchid plant parasites grows, it may be possible to refine the interpretation of DOG box presence and clustering and also develop understanding of the control of gland cell expression of effectors in other taxa.

\section{Conclusions}

The interactions between plant-parasitic nematodes and their hosts are both complex and specific. In a successful interaction, the nematodes can avoid induction of an effective host immune response, resist any immune response that is expressed and manipulate the host's developmental and cell biology to induce and maintain a functional feeding site. These interactions are mediated by an armoury of effectors that plant-parasitic nematodes appear to have assembled from adaptation of endogenous genes and also loci acquired by horizontal gene transfer from a diverse range of other taxa. To probe and understand these interactions, genomic analyses complement more directed studies, to drive and focus future programmes. Genomics can deliver whole-system analyses that permit global recovery of likely actors in parasite-host interactions. In turn, these insights can suggest new approaches to the understanding of pathogenesis and ultimately control of parasite-induced crop losses. The expanded effector set, including new effector types, the association of presence of particular effector loci with breaking of plant resistance and the definition of shared transcriptional control systems we have reported here from genomic and transcriptomic analyses of G. rostochiensis are demonstrations of this utility.

\section{Methods}

\section{Nematode culture and DNA isolation}

G. rostochiensis populations Ro1, Ro2, Ro3, Ro4 and Ro5 from the JHI PCN collection were maintained on a mixture of susceptible varieties in glasshouse conditions. For the reference assembly (Ro1), DNA was extracted according to described methods [22]. For population re- sequencing, DNA extraction was carried out as previously described [42].

\section{Genome sequencing and assembly}

Three sequencing libraries were prepared from total genomic DNA (Additional file 23: Table S11). A PCRfree 400-550 bp paired-end Illumina library was prepared using a previously described protocol [51], with the addition of sample clean up and size selection with Agencourt AMPure XP. DNA was precipitated onto beads after each enzymatic stage with an equal volume of $20 \%$ Polyethylene Glycol 6000 and $2.5 \mathrm{M}$ sodium chloride solution. Beads were not separated from the sample throughout the process until after the adapter ligation stage: fresh beads were then used for size selection. Two mate pair libraries with $\sim 2 \mathrm{~kb}$ virtual insert size were constructed [52]. The libraries were denatured using $0.1 \mathrm{M}$ sodium hydroxide and diluted to $8 \mathrm{pM}$ in hybridisation buffer for cluster amplification on the Illumina cBOT using the V3 cluster generation kit following the manufacturer's protocol, followed by a SYBRGreen cluster density QC prior to paired-end 100 base sequencing on an Illumina HiSeq2000. Raw data were analysed using the Illumina RTA1.8 analysis pipelines.

An initial assembly was produced from a combination of short-fragment paired-end and mate-pair Illumina libraries (Additional file 23: Table S11). Short paired-end sequence reads were first corrected and initially assembled using SGA v0.9.7 30 [53]. This draft assembly was then used to calculate the distribution of k-mers for all odd values of $\mathrm{k}$ between 41 and 81, using GenomeTools v.1.3.7 [54]. The k-mer length for which the maximum number of unique $k$ mers were present in the SGA assembly $(k=63)$ was then used as the $k$-mer setting for de Bruijn graph construction in a second assembly with Velvet v1.2.03 32 [55]. The mate-pair library was then used to further scaffold this Velvet assembly using SSPACE [56] with an iterative approach, in which the number of read-pair links required to scaffold two contigs was initially set to 50, then reduced to 30,20 and finally set to 10 for two final iterations of SSPACE to produce assembly nGr.v0.9. The three whole genome sequencing libraries were subsequently used to gap fill the assembly (GapFiller v1.10 [57], 10 iterations and default values for extension parameters), producing the final assembly nGr.v1.0.

A BlobDB (Blobtools v0.9.9 (https://drl.github.io/blobtools/) [58, 59] was constructed using: (1) the assembly; (2) similarity search results against the NCBI Nucleotide database (BLASTn 2.3.0+ [60] megablast, E-value cutoff $1 \mathrm{e}^{-65}$ ), Uniref90 (Diamond v0.7.12 [61], blastx, using the options -sensitive, -k 25 and -c 1) and the G. pallida 
A

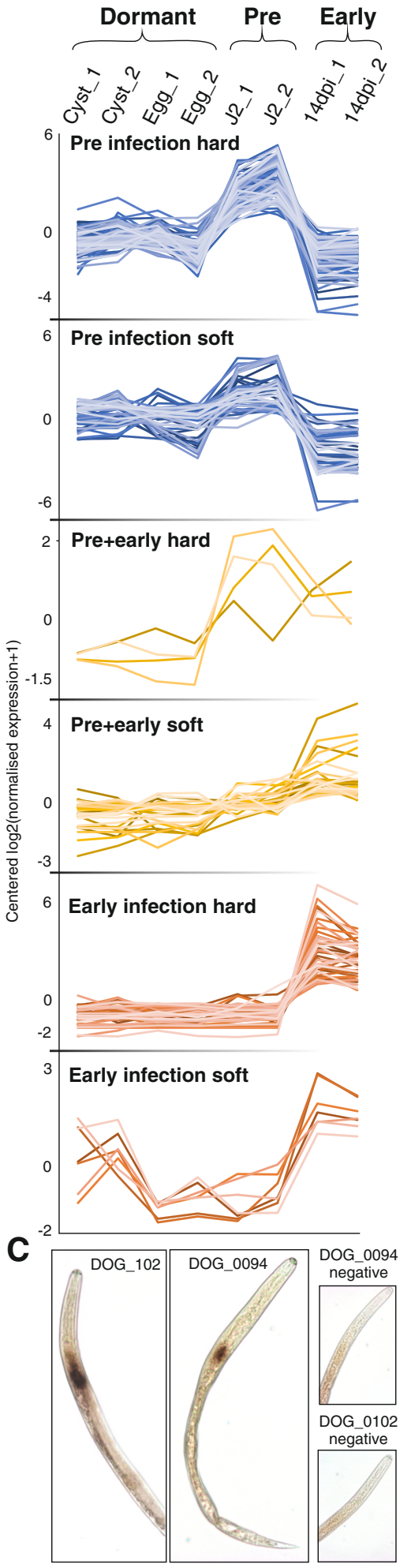

B
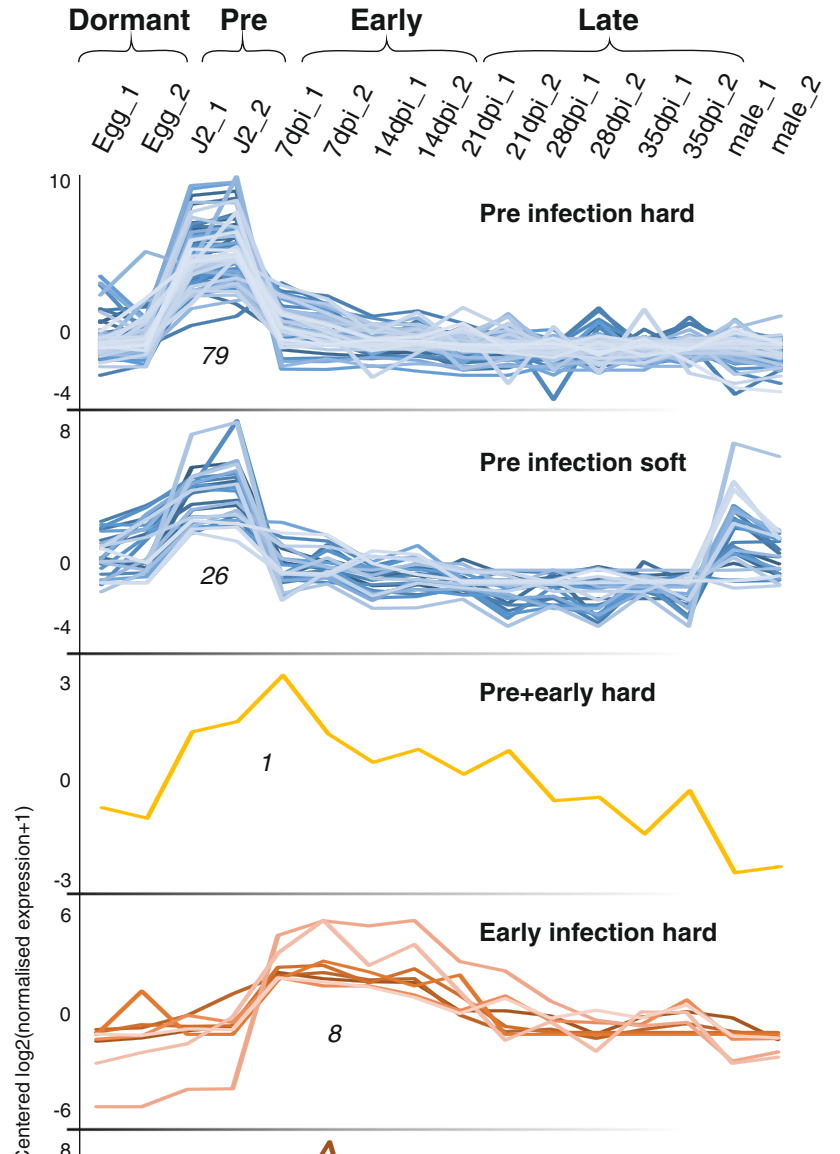

ठू

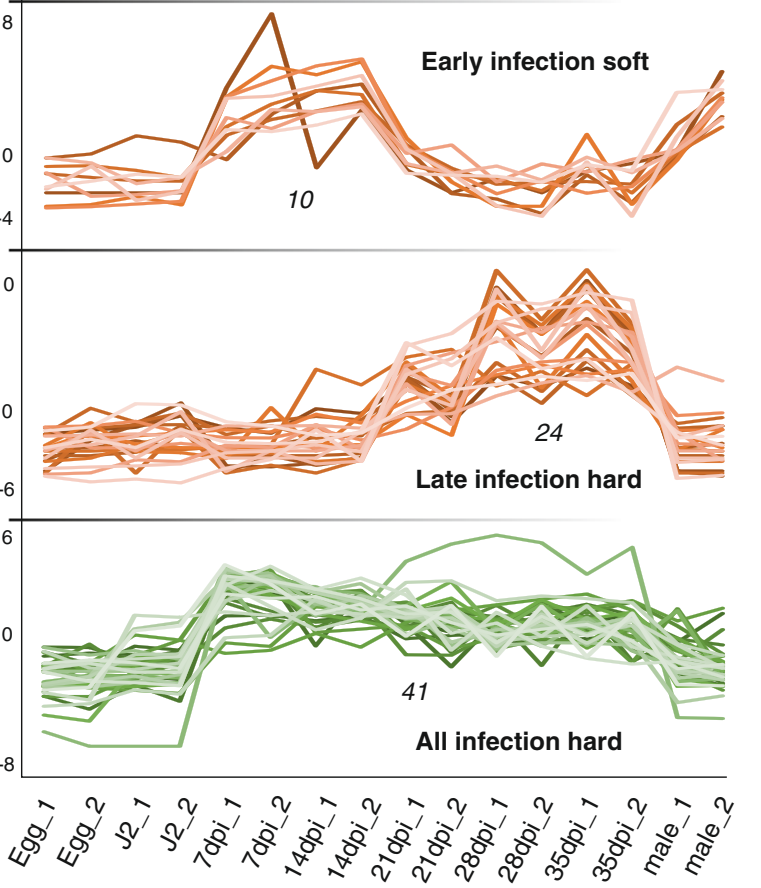

Fig. 9 (See legend on next page.) 
(See figure on previous page.)

Fig. 9 All DOG effectors from G. rostochiensis and G. pallida. Using a minimum of two DOG boxes, presence of a signal peptide, absence of transmembrane domains and temporal expression profiles consistent with a role in parasitism as selection criteria, we identify and separate all DOG effectors from G. rostochiensis (a) and G. pallida (b) into putative functional groups. For both (a) and (b), clusters were manually assigned to 'strict' or 'inclusive' subsets of the same overall expression pattern, based on how they conform to the observed pattern. $\mathbf{c}$ Experimental validation of two novel G. rostochiensis DOG effectors confirms the dorsal gland cell expression prediction. DOG_0102 (g04707) contains five DOG boxes in its promoter while DOG_0094 (g14226) contains six boxes

reference genome nGp.v1.0 (BLASTn megablast, E-value cutoff $\left.1 \mathrm{e}^{-65}\right)$; (3) the three DNA-seq read libraries mapped back to the assembly (CLC mapper v4.21.104315 CLCBio, Copenhagen, Denmark). A Taxon-Annotated-Gc-Coverage plot (TAGC) was drawn at the rank of phylum and under taxrule 'bestsum'. Using Blobtools view, taxonomically annotated nonnematode scaffolds with a bit-score $\geq 200$ were inspected manually and compared against NCBI Nucleotide database (BLASTn). Twenty-three scaffolds could be excluded as contaminants based on strong similarity to Bacteria or Fungi (span $=98.2 \mathrm{~kb}$ ). TAGC plots pre- and post-filtering are shown in Additional file 24: Figure S11. SSU/LSU rDNA screening was carried out through sequence similarity searches (BLASTn megablast) of the assembly against SILVA SSUParc and LSUParc databases. Hits were only observed against G. rostochiensis SSU (scaffolds GROS_00919, GROS_01231) and LSU (scaffold GROS_00803, GROS_00919, GROS_01231).

\section{Genome annotation}

Genome annotation was carried out in a two-step process detailed in the Additional file 2: Supplementary information. An initial round of automated gene predictions (nGr.v0.9.auto, 13,650 models) were refined in the collaborative genome annotation editor WebApollo (v1.0.4-RC3 [62]). Approximately one-eighth of the gene models were manually inspected based on homology to known Globodera genes, RNA-seq evidence and WGS read coverage yielding 1566 manually curated gene models (nGr.v0.9.manual). A second round of de novo gene prediction was carried out on assembly nGr.v1.0 with the addition of manual annotations as protein homology evidence and mapped RNA-seq reads as intronhints to train and run Augustus (v3.1 [63]) resulting in the final gene set nGr.v1.0 containing 14,309 proteincoding genes. Functional annotation was performed using InterProScan5 (v5.7-48.0 [64]) to identify motifs and domains in the proteins by comparing them against databases Gene3D, PRINTS, Pfam, Phobius, ProSitePatterns, ProSiteProfiles, SMART, SUPERFAMILY, SignalP_EUK, TIGRFAM, TMHMM, Annot8r with KEGG, GO, EC, tRNAscan and rfam. GO-Term annotation and GO-enrichment analysis was carried out using Blast2GO 3.1 .3 [65].

\section{Splicing}

Splice sites were extracted from the genomes and GFF3 files present on WormBase for the species in Additional file 4: Figure S2, using custom script extractRegionFromCoordinates.py (https://github.com/ DRL/GenomeBiology2016_globodera_rostochiensis/GNU GENERAL PUBLIC LICENSE). Four base pairs up and downstream of the $5^{\prime}$ donor site, and 6 bp upstream of the $3^{\prime}$ acceptor site were used to construct a consensus sequence for all GC/AG introns, and an identical sized sample of randomly selected GT/AG introns, using MEME SUITE v4.9.1 [66].

\section{Transcriptome sequencing and differential expression}

RNA from two life stages (hatched second-stage juvenile and 14 dpi female) was sequenced, each in biological duplicate, with Illumina Hiseq $100 \mathrm{bp}$ paired-end reads (SRA accessions ERR202479, ERR202487 and PRJEB 12075). These were compared with two additional life stages (dormant cysts and hydrated eggs), similarly sequenced in biological duplicate (Genbank accessions SAMN03393004 and SAMN03393005). All RNA-seq was carried out on pathotype Ro1. Normalized gene expression values and differentially expressed genes were identified as previously described [50]. In brief, raw reads were trimmed of adapter sequences and low quality bases (Phred $<22$, Trimmomatic [67]), mapped to the genome (Tophat2, [68]), counted on a per gene basis (bedtools v2.16.2 [69]), TMM normalised and differential expression analysis and clustering were performed using a Trinity wrapper pipeline and associated scripts for RSEM [70] and EdgeR [71] (FDR <0.001, minimum foldchange 4, [72]). Expression clusters were grouped based on the tree height parameter (12 \%) and manually assigned to expression super-clusters.

\section{Phylogenetic analysis of WAGO proteins}

Putative G. rostochiensis $(\mathrm{n}=23), \mathrm{G}$. pallida $(n=18)$ and M. hapla $(\mathrm{n}=18)$ WAGOs present in OrthoMCL clusters, which contained at least one G. rostochiensis protein with highest similarity to C. elegans WAGO1, were aligned to 545 WAGO sequences from Buck and Blaxter, 2013 [30]. This comprised WAGOs from Clade I, Clade III, Clade IV and Clade V nematodes, as well as nonNematode argonaute sequences (http://datadryad.org/ 
resource/doi:10.5061/dryad.5qs11). Alignment was carried out using clustal-omega 1.2.0 [73] and alignment was trimmed to only include the core PIWI PAZ domain section of argonautes. The WAG $+\mathrm{G}+\mathrm{F}$ model of amino acid sequence evolution was selected under AICC using Prottest 3.4 [74] and phylogenetic trees were inferred using RAxML 8.1.20 [75] (ML search + 100 rapid bootstraps).

\section{Horizontal gene transfer}

Candidate horizontal gene transfers (HGT) were detected as previously for plant-parasitic nematodes [37] by calculating AIs as described in [32, 33] using Alienness [31]. Briefly, AIs were calculated for each G. rostochiensis protein returning at least one similar sequence in either a metazoan or non-metazoan species (E-value threshold of $1 \mathrm{e}^{-3}$ ) present in NCBI's non-redundant (nr) database, according to the following formula:

$$
\begin{gathered}
A I=\log \left(\text { best metazoan } e_{\text {value }}+e^{-200}\right)-\log \\
\left(\text { best non metazoan } e_{\text {value }}+e^{-200}\right)
\end{gathered}
$$

Sequences derived from species under NCBI Taxonomy's 'Tylenchida' (TaxID: 6300, equivalent to Tylenchomorpha) were not included in this calculation to allow detection of HGT events which took place in an ancestor of cyst nematodes and their tylenchomorph relatives. No AI value could be calculated for proteins returning no similar sequences in the $\mathrm{nr}$ database. An AI $>0$ indicates a better hit to a non-metazoan species than to a metazoan species and thus a possible acquisition via HGT. An AI $>30$ corresponds to a difference of magnitude $\mathrm{e}^{10}$ between the best non-metazoan and best metazoan $\mathrm{E}$-values and is estimated to be a strong indication of a HGT event [32]. Proteins with an AI $>0$ and $\geq 70 \%$ identity to a non-metazoan protein were considered putative contaminants and not included in further analysis.

\section{Effector identification}

Genes in the G. rostochiensis genome sequence similar to previously reported effectors with experimentally validated gland cell expression were identified in a two-step process. An inclusive list of effectors was generated by sequence similarity alone. For those effectors that are characterised by the presence of particular domains (e.g. the SPRY domain of SPRY-SEC effectors), hmmsearch [76] using the appropriate domain was used to identify all sequences predicted to contain the same domain using the gathering significance threshold. For all other effectors, BLASTp was used to identify similar sequences (E-value $\leq 1 \mathrm{e}^{-5}$ ). Cell wall degrading enzymes (CWDEs) identified as putatively acquired via HGT were included if they had known in situ localisation to either gland cell. This inclusive list was triaged by removing those without a predicted signal peptide and/or those with one or more transmembrane domain (Phobius [77]), producing the high-confidence effector list (Additional file 12: Table S4).

\section{Variant analysis}

Sequence reads (Bioproject PRJNA305631) were mapped against the assembly using bwa mem v0.7.12-r1044 [78]. Duplicated read pairs were removed using Picard (http:// broadinstitute.github.io/picard). Variants were called using freebayes v0.9.20-16-g3e35e72 [79]. Haplotypes and other complex variants were decomposed using vcflib vcfallelicprimitives v1.0.0-rc0 (https://github.com/ekg/vcflib/releases/tag/v1.0.0-rc0) followed by normalisation using vt normalize v0.57 [80]. The resulting VCF file was filtered with the following parameters: DP $>10$ \& $\mathrm{MQM}>30$ \& $\mathrm{QUAL}>1$ \& $\mathrm{QUAL} / \mathrm{AO}>10$ \& $\mathrm{SAF}>2 \& \mathrm{SAR}>2 \&$ RPR $>1$ \& RPL $>1$ using vcffilter from vcflib. Variants were annotated using SnpEff v4.1 L [81]. The resulting VCF file was analysed using vt peek, RTG Tools [82] and parse_snpeff.py. Variants (vcf file) were filtered to retain only SNPs $($ TYPE $=$ snp) with no missing data, 730,705 loci were found from whole genome data. Allele frequencies at each locus was computed by dividing the reference allele observation count (RO) by the read depth (DP). In the same manner, allele frequencies for SNPs present in non-coding regions $(n=619,886)$ were computed. Seqboot module in PHYLIP v3.695 [83] was used to make 100 bootstrapped datasets. Maximum likelihood phylogenetic trees of the nine populations of G. rostochiensis were calculated with the Contml module based on genome-wide SNP allele frequencies and a majority rule consensus tree was constructed using Consense. Principal component analysis (PCA) were calculated with the prcomp() function from the stats package in $\mathrm{R}$ based on genome-wide allele frequencies at these 730,705 loci.

\section{Protein clustering}

Putative one-to-one orthologues between G. pallida and G. rostochiensis were identified by the reciprocal best BLAST hit method. Both proteomes were compared against each other using BLASTp (v2.2.30+) and the resulting files were processed using the script rbbh.py (https://github.com/DRL/GenomeBiology2016_globodera_rostochiensis GNU GENERAL PUBLIC LICENSE, Evalue $\leq 1 \mathrm{e}^{-25}$ and reciprocal-query coverage $>75 \%$ ). Protein clustering analysis was performed on the proteomes (retrieved from Wormbase WS248) of B. xylophilus, C. elegans, M. hapla, M. incognita, G. pallida (retrieved from WormBase ParaSite WBPS2) and G. rostochiensis (nGr.v1.0) using OrthoMCL (v2.0.9 [84]) (with an inflation value of 1.5) and following the guidelines specified in [84]. Phylogenetically informative sets of clusters were plotted using UpSetR (Release v1.0.0, https://github.com/hms- 
$\mathrm{dbmi} / \mathrm{UpSetR} /$ releases [85]). For each of four orthologous gene cluster categories (all nematodes tested, all plant parasites tested, Globodera and Meloidogyne and Globodera alone), the percentage of genes present in each differential expression super-cluster was determined. This value was normalised by the total number of genes present in each given differential expression super-cluster, to return a relative measure of abundance used in Fig. 3.

\section{Effector islands, synteny and promoter analyses}

The presence of effectors in adjacent $(n \pm 1)$, or neighbouring positions (up to \pm 9 ), was determined. As a negative control, a subset of 612 G. rostochiensis gene families not predicted to contain effectors was identified from the OrthoMCL. This subset contained gene families of various sizes, the distribution of which with respect to gene family size 1,2 and $\geq 3$ was the same as that of the effectors. Starting from this initial negative set of 612 gene families, 37 were selected at random and the presence of genes from these 37 families in adjacent ( $\mathrm{n} \pm 1$ ), or neighbouring positions (up to \pm 9 ), was determined. This process was repeated for 1000 iterations to generate a robust negative for the average frequency in each neighbouring position. The observed frequency of effector occurrence at each position was compared to the average of 1000 iterations. Non-overlapping islands, delineated by furthest distance at which statistically significant enrichment was observed $\left( \pm 6, X^{2}\right.$ goodness of fit, $p<0.001$ ), were manually identified.

Synteny between scaffolds of G. pallida and G. rostochiensis was assessed based on OrthoMCL-cluster membership of both sets of proteins using i-adhore-3.0.01 ((https:// github.com/widdowquinn/Teaching/tree/master/Comparative_Genomics_and_Visualisation/Part_2/i-ADHore) type = family, tandem_gap $=10$, gap_size $=15$, max_gaps_in_alignment $=20$, cluster_gap $=20$, q_value $=0.9$, alignment method $=$ gg2, prob_cutoff $=0.001$, multiple_hypothesis _ correction $=$ bonferroni, anchor_points $=5$ ). Syntenic blocks were visualised as clusters in a graph using parse_iadhore.py. G. rostochiensis scaffold GROS_00007 (a member of the biggest syntenic cluster) was plotted with its homologous G. pallida scaffolds using circos $0.67-7$, including GCcontent and BLASTn results at an E-value cutoff of 1e-65.

To analyse putative enhancer elements, sequences $500 \mathrm{bp}$ upstream of genes of interest (termed the promoter regions) were extracted from the genome using get_upstream_regions.py (https://github.com/peterthorpe5 GNU GENERAL PUBLIC LICENSE). Enrichment of motifs between categories (DG versus all, DG versus SvG, etc.) was calculated using HOMER [49], specifying max length of six nucleotides. Instances of the motif were identified in FASTA sequences of promoter regions using the FIMO web server [86].

\section{In situ hybridisation}

The spatial expression patterns of two predicted G. rostochiensis dorsal gland effectors were determined in J2s by in situ hybridisation as described previously [87]. Singlestranded digoxygenin-labelled DNA probes were synthesised from amplified cDNA fragments using primers g14226F (5'-CCGTTGAGCCGTCGACTAAT-3') and g1 4226R (5'-TTTCCCGACGTCCAGTTGAC-3') or g0470 7F (5'-AAGGAGCACCATCGTACCAAG-3') and g04707 R (5'-GTTCTGAGCCTTGTTGAAAG-3').

\section{Description of additional data files}

The following additional data are available with the online version of this paper. Additional file 7: File S1 contains the data matrix of normalised expression values. Additional file 2: Supplementary information file 1 contains various supplemental methods and results.

\section{Additional files}

Additional file 1: Figure S1. Gene duplication in the G. rostochiensis and related genomes. Comparing the identity of each gene to the next most similar gene in the genome gives insights into potential duplication within the genome sequence. In a diploid species, with a good assembly and gene prediction, we expect no overrepresentation of duplicates at any particular divergence, as is seen in the genome of M. hapla. The G. rostochiensis protein set has a very similar distribution to that of M. hapla, but in G. pallida there is an overrepresentation of genes that are $>97 \%$ identical to each other. As reported previously, the protein set from $M$. incognita has a distinct excess of duplicates at $~ 96 \%$ identity, thought to derive from a hybrid origin, and subsequent aneuploidy changes, of this species $[23,88]$. G. pallida is not believed to derive from a hybridisation event [26] and so this is probably a reflection of duplication at the assembly stage (i.e. retention of allelic copies of loci because of the high level of heterozygosity in UK populations). (PDF $1365 \mathrm{~kb}$ )

Additional file 2: Supplementary methods and results. (DOCX 28 kb)

Additional file 3: Table S1. Genome annotation. (XLSX 9 kb)

Additional file 4: Figure S2. Phylum wide analysis of GC/AG splice sites in nematodes. The percentage of GC/AG splices sites with associated consensus sequences are shown for 17 species against a schematic phylogeny of the phylum Nematoda (adapted from [89]). Red numbers indicate those which likely represent under reporting due to over-strict parameter settings during gene prediction. (PDF 1371 kb)

Additional file 5: Figure S3. Comparison of gene expression between parasitic G. pallida life stages. The key transitions to be captured in terms of gene expression of all genes, and in particular for effectors, is from outside the plant (J2) compared to inside the plant (sedentary female). There is almost no difference in global gene expression between the early sedentary time points [22]. A. $84 \%$ of the variation in expression at 7 days post infection (dpi) is explained by variation in expression at 14 dpi. B. This correlation is even more profound if the analysis is restricted to only the effectors (89\%). Similar correlations are possible between 14 and $21 \mathrm{dpi}$, albeit of lesser magnitude but an identical trend (60\% correlation for all genes, and $64 \%$ correlations for specifically effectors). This is not the case, however, when comparing $J 2$ and $7 \mathrm{dpi}$ (44\% for all genes, and zero correlation for all effectors). Fourteen dpi provides an ideal intermediate for the sedentary stages. (PDF 4379 kb)

Additional file 6: Figure S4. Differential expression super-clusters. Ninety-four percent of all differentially expressed genes are manually grouped into 25 biologically relevant expression super-clusters. For each super-cluster, individual cluster graphs are shown where for all expression 
displayed as centred log fold-change is in the order, Cyst, Cyst, Egg, Egg, J2, J2, 14 dpi, 14 dpi. (PDF $1669 \mathrm{~kb}$ )

Additional file 7: File S1. Clusters and normalised expression tables. (XLSX $956 \mathrm{~kb})$

Additional file 8: Figure S5. Phylogenetic analysis of Worm-specific Argonauts (WAGOs). A. A total of 604 nematode and non-nematode argonaute proteins drawn as an unrooted phylogram. It is assumed that each subtree $(B-F)$ is effectively rooted by the other subtrees; however, the extreme divergence between these proteins yields low support for some subtrees. B-F Subtrees containing G. rostochiensis WAGOs (GrWAGOs). Branch widths of subtrees are drawn proportional to branch support. Coloured boxes indicate membership of taxa to phylogenetic groups (nematode Clade I, Clade III, Clade IV and Clade V, non-nematode taxa) and coloured stars indicate clades composed entirely of HeteroderidaeWAGOs (Globodera spp., Meloidogyne spp., Heterodera glycines). Orange dots indicate GrWAGOs in differential expression super-clusters 19, 20 or 21. GrWAGOs are placed within Nematode WAGO-subclades ALG1/ ALG2 (1), RDE1/ERGO1/PRG1/2 (2), WAGO1/2/4/5 (7), CSR/WAGO-III/ WAGO-IV (8) and NRDE/WAGO-10/11 (5), sensu Buck and Blaxter, 2013 [30]. As expected, no GrWAGOs were observed in WAGO-subclades SAGO2/PPW, WAGO-III/-V and ALG3/ALG4. Subclades NRDE/WAGO-10/ 11, WAGO1/2/4/5 and CSR/WAGO-III/WAGO-IV show increased numbers of paralogous expansion of these gene families within Clade IV in general and Heteroderidae in particular. B All Globodera spp. and Meloidogyne spp. exhibit one ALG1/ALG2 orthologue each, which form a clade. C The RDE1/ERGO1/PRG1/2 subtree contains another Heteroderidae-specific clade; however, one $\mathrm{M}$. incognita sequence is sister to a subclade of Clade III taxa. This is surprising since the Clade III parasites A. suum and B. pahangi are thought to have lost the piRNA pathway [30] and may be an artefact. D The NRDE/WAGO-10/ WAGO-11 subtree shows an expansion of paralogous HeteroderidaeWAGOs, with three GrWAGOs expressed at 14 dpi. E The WAGO1/2/4/ 5 subtree depicts two Heterodera-specific expansions, of which the larger subclade contains four GrWAGOs expressed at $14 \mathrm{dpi}$. F The CSR/WAGO-III/WAGO-IV subtree contains another two Heteroderidaespecific expansion, of which one also includes a sequence from the Clade V nematode Dictyocaulus viviparus (ochre star). (PDF 64 kb)

Additional file 9: Table S2. Alien indices. (XLSX $81 \mathrm{~kb}$ )

Additional file 10: Table S3. High confidence effectors. (XLSX $13 \mathrm{~kb}$ )

Additional file 11: Figure S6. Transposable elements in G. rostochiensis, G. pallida and Meloidogyne spp. Quantities of transposable elements from (A) DNA, (B) LTR, (C) LINE and (D) SINE super-familes. Globodera spp. contain notably more Jockey (LINE) and Alu (SINE) than the Meloidogyne spp. (PDF $623 \mathrm{~kb}$ )

Additional file 12: Table S4. Genes acquired via HGT in other cyst and root-knot nematodes also found in the genome of $G$. rostochiensis. (XLSX $21 \mathrm{~kb})$

Additional file 13: Table S5. Variants summary. (XLSX $11 \mathrm{~kb}$ ) Additional file 14: Table S6. Molecular markers of pathotypes Ro1, 4 and 5. (XLSX $24 \mathrm{~kb})$

Additional file 15: Figure S7. Phylogenetic analysis of G. rostochiensis pathotypes. A. A maximum likelihood phylogeny based on 730,705 genome wide SNPs. Two distinct groups of Ro1 are together separated from Ro2, Ro3, Ro4 and Ro5. Node labels indicate bootstrap support values for 100 iterations. B. Principle component analysis (PCA) based on the same dataset suggest that for pathotype Ro1, intra-pathotype variation is similar to inter-pathotype variation. REF Ro1 $=$ Reference strain Ro1 assembly, MAR149 Ro1 = British Columbia, QC Ro1 = Quebec, Ro19 Ro1 = European, NFLD Ro1 = Newfoundland, SCRI_Ro2, SCRI_Ro3, SCRI_Ro4, SCRI_Ro5 are pathotype populations from the James Hutton Institute collection. (PDF $1636 \mathrm{~kb}$ )

Additional file 16: Table S7. SNP allele frequency correlation with virulence in non-effectors. (XLSX $15 \mathrm{~kb}$ )

Additional file 17: Figure S8. Comparison of distance to neighbouring gene for effectors and non-effectors. A. The log nucleotide distance to next gene $5^{\prime}$ and $3^{\prime}$ for a random subset of non-effectors $(n=138)$. B. The $\log$ nucleotide distance to next gene $5^{\prime}$ and $3^{\prime}$ for high-confidence effectors $(n=138)$. C. Comparing the distance of each gene to its neighbour either upstream or downstream suggests that despite being located in gene dense regions of the genome, effectors have a skewed distribution of gene density compared to an identically sized subset of non-effectors (lower case letters indicate homogenous subsets, Student's t-test correcting for multiple comparisons $p<0.001)$. (PDF $1393 \mathrm{~kb}$ )

Additional file 18: Table S8. Effector islands. (XLSX $13 \mathrm{~kb}$ )

Additional file 19: Figure S9. Synteny clusters. Network representation of i-ADHoRe results. Scaffolds are represented as nodes (blue $=G$. rostochiensis, ochre $=G$. pallida) whose diameter is scaled by scaffold length and vertices are drawn between them if five or more anchorpoints (i.e. syntenic proteins, numbers on vertices indicate count of anchorpoints) have been found. The biggest cluster (dashed black line) is composed of $26 \mathrm{G}$. rostochiensis scaffolds (4.99 Mb) and $35 \mathrm{G}$. pallida scaffolds (5.54 Mb). Part of this cluster (grey dotted line) is shown in Fig. 6. (PDF $570 \mathrm{~kb}$ )

Additional file 20: Figure S10. The DOG box is not a quantitative predictor of temporal expression. A. In G. pallida, genes with four or more motifs and a signal peptide are primarily expressed during the infective stage. Each line represents the expression pattern of a different gene. B. The number of motifs does not correlate with gene expression at J2 (black, all numbers of motifs; red, four or more motifs). (PDF $1707 \mathrm{~kb}$ )

Additional file 21: Table S9. G. rostochiensis putative DOG effectors. (XLSX $36 \mathrm{~kb}$ )

Additional file 22: Table S10. G. pallida putative DOG effectors. (XLSX $44 \mathrm{~kb}$ )

Additional file 23: Table S11. G. rostochiensis genome libraries. (XLSX $9 \mathrm{~kb}$ )

Additional file 24: Figure S11. Blob plots. A. Blob plot of the initial G. rostochiensis assembly, displaying some minor contamination from Actinobacteria and Ascomycota. Each scaffold is drawn as a circle based on its GC content (X-axis) and log-coverage ( $Y$-axis), with a diameter proportional to its length and coloured by its taxonomic annotation at the phylum-level. In the legend, colours of phyla are listed together with scaffold-count, scaffold span and scaffold N50. The histograms above and to the right of the main scatter plot sum contig spans for GC proportion bins and log-coverage bins, respectively. B. Blob plot of the G. rostochiensis assembly after removal of contaminant scaffolds. (PDF $8314 \mathrm{~kb}$ )

Additional file 25: Table S12. Microsatellite abundance. (XLSX 8 kb)

\section{Acknowledgements}

This work benefited from interactions promoted by COST Action project FA 1208. We thank Nathalie Smerdon and Lesley Shirley for creating the sequencing libraries and Matthew Berriman for supporting this work. The authors are grateful to the Genotoul bioinformatics platform Toulouse Midi-Pyrenees for providing computing resources.

\section{Funding}

SE-vdA is supported by BBSRC grant BB/M014207/1. Sequencing was funded by BBSRC grant BB/F000642/1 to the University of Leeds and grant BB/ F00334X/1 to the Wellcome Trust Sanger Institute). DRL was supported by a fellowship from The James Hutton Institute and the School of Biological Sciences, University of Edinburgh. GK was supported by a BBSRC PhD studentship. The James Hutton Institute receives funding from the Scottish Government. JAC and NEH are supported by the Wellcome Trust through its core funding of the Wellcome Trust Sanger Institute (grant 098051). This work was also supported by funding from the Canadian Safety and Security Program, project number CRTI09_462RD.

\section{Availability of data and materials}

Genome sequence data are available in the SRA repository accessions ERR114519, ERR123958 and ERR114520. Transcriptomic data are available in the SRA repository under accession ERR202479, ERR202487 and PRJEB12075 and the GenBank repository accessions SAMN03393004 and SAMN03393005. Whole genome resequencing data are available under the Bioproject PRJNA305631. Raw and parsed VCF files, transposable element prediction and gene coordinates, clusters and normalised expression tables, interproscan results and blast2go results are available in Dryad accession doi:10.5061/dryad.455r6. Custom scripts integral to the manuscript are 
available on GitHub and cited where appropriate in the text. The G. rostochiensis genome is available for query (BLAST etc) at: http:// globodera.bio.ed.ac.uk/search/species_search?genomeSelect=1213186 $\&$ Gid=1213187\&GFFid=1213188).

\section{Authors' contributions}

NEH and JAC carried out sequencing and assembly. DRL and GK carried out automated annotations and gap filling. SEVDA, DRL, EGJD, BM, EG, MOD, IB, $J$, ME, MS, MR, HO, AT, GS, SM, PJAC, WP, CJL, JMCM, LMJ, JTJ and HBY participated in manual annotation. DRL and SEVDA carried out phylum and genus comparative analyses. DRL, BM, MOD, IB and SEVDA carried out variant analysis. JM carried out microsatellite analysis. SEVDA and MDR carried out transcriptomic analyses. EGJD and CR carried out HGT calculations and analyses. BH carried out carbohydrate-active enzymes analyses. AS predicted transposable elements. SEVDA and DRL carried out splicing analyses. SEVDA, PT and CJL carried out effectors analyses. CJL carried out in situ hybridisation. SEVDA, DRL, EGJD, MB and JTJ wrote the manuscript. VB, PEU and MB critically revised the manuscript. SEVDA, DRL, JTJ, CJL, VB, PEU and MB participated in design of the study. All authors read and approved the final manuscript.

\section{Competing interests}

The authors declare that they have no competing interests.

\section{Ethics approval and consent to participate}

Ethics approval was not needed for the study.

\section{Author details}

${ }^{1}$ Division of Plant Sciences, College of Life Sciences, University of Dundee, Dundee DD1 5EH, UK. ${ }^{2}$ Institute of Evolutionary Biology, University of Edinburgh, Edinburgh EH9 3FL, UK. ${ }^{3}$ Cell and Molecular Sciences Group, Dundee Effector Consortium, James Hutton Institute, Dundee DD2 5DA, UK. ${ }^{4}$ Centre for Plant Sciences, University of Leeds, Leeds LS2 9JT, UK. ${ }^{5}$ INRA, University Nice Sophia Antipolis, CNRS, UMR 1355-7254 Institut Sophia Agrobiotech, 06900 Sophia Antipolis, France. ${ }^{6}$ Wellcome Trust Sanger Institute, Wellcome Trust Genome Campus, Cambridge CB10 1SA, UK ${ }^{7}$ School of Biological, Biomedical and Environmental Sciences, University of Hull, Hull HU6 7RX, UK. ${ }^{8}$ INRA, UMR1349 IGEPP (Institute for Genetics, Environment and Plant Protection), 35653 Le Rheu, France. ${ }^{9}$ Agriculture and Agri-food Canada, Horticulture Research and Development Centre, 430 Bboul. Gouin, St-Jean-sur-Richelieu, Quebec J3B 3E6, Canada. ${ }^{10}$ Sidney Laboratory, Canadian Food Inspection Agency (CFIA), 8801 East Saanich Rd, Sidney, BC V8L 1H3, Canada. " 1 Laboratory of Nematology, Department of Plant Sciences, Wageningen University, Droevendaalsesteeg 1, 6708, PB, Wageningen, The Netherlands. ${ }^{12}$ Information and Computational Sciences Group, James Hutton Institute, Dundee, UK. ${ }^{13}$ USDA-ARS Horticultural Crops Research Laboratory, Corvallis, OR, USA. ${ }^{14}$ CNRS UMR 7257, INRA, USC 1408, Aix-Marseille University, AFMB, 13288 Marseille, France. ${ }^{15}$ Department of Biological Sciences, King Abdulaziz University, Jeddah, Saudi Arabia. ${ }^{16}$ School of Biology, University of St Andrews, North Haugh, St Andrews KY16 9TZ, UK.

\section{Received: 8 January 2016 Accepted: 12 May 2016}

Published online: 10 June 2016

\section{References}

1. Nicol JM, Turner SJ, Coyne DL, den Nijs L, Hockland S, Maafi ZT. Current nematode threats to world agriculture. In: Jones J, Gheysen G, Fenoll C, editors. Genomics and molecular genetics of plant-nematode interactions. The Netherlands: Springer; 2011. p. 21-43.

2. Evans K, Franco J, De Scurrah MM. Distribution of species of potato cystnematodes in South America. Nematologica. 1975;21:365-9.

3. Plantard O, Picard D, Valette S, Scurrah M, Grenier E, Mugniery D. Origin and genetic diversity of Western European populations of the potato cyst nematode (Globodera pallida) inferred from mitochondrial sequences and microsatellite loci. Mol Ecol. 2008;17:2208-18.

4. Evans K, Rowe J. Distribution and economic importance. In: Sharma SB, editor. The cyst nematodes. The Netherlands: Springer; 1998. p. 1-30

5. Boucher AC, Mimee B, Montarry J, Bardou-Valette S, Bélair G, Moffett P, et al. Genetic diversity of the golden potato cyst nematode Globodera rostochiensis and determination of the origin of populations in Quebec, Canada. Mol Phylogenet Evol. 2013;69:75-82.
6. Phillips M, Trudgill D. Variation of virulence, in terms of quantitative reproduction of Globodera pallida populations, from Europe and South America, in relation to resistance from Solanum vernei and S. tuberosum ssp. andigena CPC 2802. Nematologica. 1998:44:409-23.

7. Hockland S, Niere B, Grenier E, Blok V, Phillips M, Den Nijs L, et al. An evaluation of the implications of virulence in non-European populations of Globodera pallida and G. rostochiensis for potato cultivation in Europe. Nematology. 2012;14:1-13.

8. Kort J, Ross H, Rumpenhorst H, Stone A. An international scheme for identifying and classifying pathotypes of potato cyst-nematodes Globodera rostochiensis and G. pallida. Nematologica. 1977;23:333-9.

9. Sobczak M, Golinowski W. Cyst nematodes and syncytia. In: Jones J, Gheysen G, Fenoll C, editors. Genomics and molecular genetics of plantnematode interactions. The Netherlands: Springer; 2011. p. 61-82

10. Thorpe P, Mantelin S, Cock PJ, Blok VC, Coke MC, Eves-van den Akker S, et al. Genomic characterisation of the effector complement of the potato cyst nematode Globodera pallida. BMC Genomics. 2014;15:923.

11. Eves-van den Akker S, Lilley CJ, Jones JT, Urwin PE. Identification and characterisation of a hyper-variable apoplastic effector gene family of the potato cyst nematodes. PLoS Pathog. 2014;10, e1004391.

12. Smant G, Stokkermans J, Yan YT, de Boer JM, Baum TJ, Wang XH, et al. Endogenous cellulases in animals: Isolation of beta-1,4-endoglucanase genes from two species of plant-parasitic cyst nematodes. Proc Natl Acad Sci U S A. 1998;95:4906-11.

13. Mei Y, Thorpe P, Guzha A, Haegeman A, Blok VC, MacKenzie K, et al. Only a small subset of the SPRY domain gene family in Globodera pallida is likely to encode effectors, two of which suppress host defences induced by the potato resistance gene Gpa2. Nematology. 2015;17:409-24.

14. Lee C, Chronis D, Kenning C, Peret B, Hewezi T, Davis EL, et al. The novel cyst nematode effector protein $19 \mathrm{CO}$ interacts with the Arabidopsis auxin influx transporter LAX3 to control feeding site development. Plant Physiol. 2011;155:866-80.

15. Wang XH, Mitchum MG, Gao BL, Li CY, Diab H, Baum TJ, et al. A parasitism gene from a plant-parasitic nematode with function similar to CLAVATA3/ ESR (CLE) of Arabidopsis thaliana. Mol Plant Pathol. 2005;6:187-91.

16. Danchin EGJ, Rosso M-N, Vieira P, de Almeida-Engler J, Coutinho PM, Henrissat $B$, et al. Multiple lateral gene transfers and duplications have promoted plant parasitism ability in nematodes. Proc Natl Acad Sci U S A. 2010;107:17651-6.

17. Haegeman A, Jones JT, Danchin EGJ. Horizontal gene transfer in nematodes: a catalyst for plant parasitism? Mol Plant-Microbe Interact. 2011;24:879-87.

18. Maier TR, Hewezi T, Peng J, Baum TJ. Isolation of whole esophageal gland cells from plant-parasitic nematodes for transcriptome analyses and effector identification. Mol Plant-Microbe Interact. 2013;26:31-5.

19. Bellafiore S, Shen Z, Rosso M-N, Abad P, Shih P, Briggs SP. Direct identification of the Meloidogyne incognita secretome reveals proteins with host cell reprogramming potential. PLoS Pathog. 2008;4:e1000192.

20. Hewezi T, Juvale PS, Piya S, Maier TR, Rambani A, Rice JH, et al. The cyst nematode effector protein 10A07 targets and recruits host posttranslational machinery to mediate its nuclear trafficking and to promote parasitism in Arabidopsis. Plant Cell. 2015:27:891-907.

21. Wang J, Replogle A, Hussey R, Baum T, Wang X, Davis EL, et al. Identification of potential host plant mimics of CLAVATA3/ESR (CLE)-like peptides from the plant-parasitic nematode Heterodera schachtii. Mol Plant Pathol. 2011;12:177-86.

22. Cotton JA, Lilley CJ, Jones LM, Kikuchi T, Reid AJ, Thorpe $P$, et al. The genome and life-stage specific transcriptomes of Globodera pallida elucidate key aspects of plant parasitism by a cyst nematode. Genome Biol. 2014;15:R43.

23. Abad P, Gouzy J, Aury J-M, Castagnone-Sereno P, Danchin EGJ, Deleury E, et al. Genome sequence of the metazoan plant-parasitic nematode Meloidogyne incognita. Nat Biotechnol. 2008;26:909-15.

24. Opperman CH, Bird DM, Williamson VM, Rokhsar DS, Burke M, Cohn J, et al. Sequence and genetic map of Meloidogyne hapla: A compact nematode genome for plant parasitism. Proc Natl Acad Sci. 2008;105:14802-7.

25. Consortium S. Genome sequence of the nematode C. elegans: a platform for investigating biology. Science. 1998;282:2012-8.

26. Grisi E, Burrows PR, Perry RN, Hominick WM. The genome size and chromosome complement of the potato cyst nematode Globodera pallida. Fundam Appl Nematol. 1995;18:67-70.

27. Burset M, Seledtsov I, Solovyev V. Analysis of canonical and non-canonical splice sites in mammalian genomes. Nucleic Acids Res. 2000;28:4364-75.

28. Farrer T, Roller AB, Kent WJ, Zahler AM. Analysis of the role of Caenorhabditis elegans GC-AG introns in regulated splicing. Nucleic Acids Res. 2002;30: 3360-7. 
29. Kikuchi T, Cotton JA, Dalzell JJ, Hasegawa K, Kanzaki N, McVeigh P, et al. Genomic insights into the origin of parasitism in the emerging plant pathogen Bursaphelenchus xylophilus. PLoS Pathog. 2011;7:e1002219.

30. Buck AH, Blaxter M. Biogenesis and turnover of small RNAs: functional diversification of Argonautes in nematodes: an expanding universe. Biochem Soc Trans. 2013:41:881.

31. Rancurel C, Da Rocha M, Danchin EGJ. Alienness: rapid detection of horizontal gene transfers in metazoan genomes. F1000Posters 2014. 5:1666 (poster).

32. Flot J-F, Hespeels B, Li X, Noel B, Arkhipova I, Danchin EG, et al. Genomic evidence for ameiotic evolution in the bdelloid rotifer Adineta vaga. Nature. 2013;500:453-7.

33. Gladyshev EA, Meselson M, Arkhipova IR. Massive horizontal gene transfer in bdelloid rotifers. Science. 2008;320:1210-3.

34. Danchin EGJ, Guzeeva EA, Mantelin S, Berepiki A, Jones JT. Horizontal gene transfer from bacteria has enabled the plant-parasitic nematode Globodera pallida to feed on host-derived sucrose. Mol Biol Evol. 2016;33:1571-79.

35. Dong S, Stam R, Cano LM, Song J, Sklenar J, Yoshida K, et al. Effector specialization in a lineage of the Irish potato famine pathogen. Science. 2014;343:552-5.

36. Gilroy EM, Breen S, Whisson SC, Squires J, Hein I, Kaczmarek M, et al. Presence/absence, differential expression and sequence polymorphisms between PiAVR2 and PiAVR2-like in Phytophthora infestans determine virulence on R2 plants. New Phytol. 2011;191:763-76.

37. Eves-van den Akker S, Lilley C, Danchin E, Rancurel C, Cock P, Urwin P, Jones J. The transcriptome of Nacobbus aberrans reveals insights into the evolution of sedentary endoparasitism in plant-parasitic nematodes. Genome Biol Evol. 2014:6:2181-94.

38. Hussey R, Mims C. Ultrastructure of esophageal glands and their secretory granules in the root-knot nematode Meloidogyne incognita. Protoplasma. 1990;156:9-18

39. Endo B. Ultrastructure of subventral gland secretory granules in parasitic juveniles of the soybean cyst nematode, Heterodera glycines. J Helminthol Soc Wash. 1993;60:22-34

40. Endo BY. Ultrastructure of esophageal gland secretory granules in juveniles of Heterodera glycines. J Nematol. 1987;19:469.

41. Maule AG, Curtis R. Parallels between plant and animal parasitic nematodes In: Jones J, Gheysen G, Fenoll C, editors. Genomics and molecular genetics of plant-nematode interactions. The Netherlands: Springer; 2011. p. 221-51.

42. Mimee B, Duceppe MO, Véronneau PY, Lafond-Lapalme J, Jean M, Belzile F, et al. A new method for studying population genetics of cyst nematodes based on Pool-Seq and genomewide allele frequency analysis. Mol Ecol Resour. 2015;15:1356-65.

43. Noon JB, Hewezi TAF, Maier TR, Simmons C, Wei J-Z, Wu G, et al. Eighteen new candidate effectors of the phytonematode Heterodera glycines produced specifically in the secretory esophageal gland cells during parasitism. Phytopathology. 2015;105:1362-72.

44. Gao BL, Allen R, Maier T, Davis EL, Baum TJ, Hussey RS. The parasitome of the phytonematode Heterodera glycines. Mol Plant-Microbe Interact. 2003;16:720-6.

45. Jones JT, Kumar A, Pylypenko LA, Thirugnanasambandam A, Castelli L, Chapman $S$, et al. Identification and functional characterization of effectors in expressed sequence tags from various life cycle stages of the potato cyst nematode Globodera pallida. Mol Plant Pathol. 2009;10:815-28.

46. Chronis D, Chen S, Lu S, Hewezi T, Carpenter SC, Loria R, et al. A ubiquitin carboxyl extension protein secreted from a plant-parasitic nematode Globodera rostochiensis is cleaved in planta to promote plant parasitism. Plant J. 2013;74:185-96.

47. Raffaele S, Win J, Cano LM, Kamoun S. Analyses of genome architecture and gene expression reveal novel candidate virulence factors in the secretome of Phytophthora infestans. BMC Genomics. 2010;11:637.

48. Vens C, Rosso M-N, Danchin EG. Identifying discriminative classificationbased motifs in biological sequences. Bioinformatics. 2011;27:1231-8.

49. Heinz S, Benner C, Spann N, Bertolino E, Lin YC, Laslo P, et al. Simple combinations of lineage-determining transcription factors prime cisregulatory elements required for macrophage and B cell identities. Mol Cell. 2010;38:576-89.

50. Espada M, Silva AC, Eves van den Akker S, Cock PJ, Mota M, Jones JT. Identification and characterization of parasitism genes from the pinewood nematode Bursaphelenchus xylophilus reveals a multi-layered detoxification strategy. Mol Plant Pathol. 2016;17:286-95.

51. Kozarewa I, Ning Z, Quail MA, Sanders MJ, Berriman M, Turner DJ. Amplification-free Illumina sequencing-library preparation facilitates improved mapping and assembly of (G+C)-biased genomes. Nat Methods. 2009;6:291-5.

52. Park N, Shirley L, Gu Y, Keane TM, Swerdlow H, Quail MA. An improved approach to mate-paired library preparation for Illumina sequencing. Methods Next Generation Seq. 2013;1:10-20.

53. Simpson JT, Durbin R. Efficient de novo assembly of large genomes using compressed data structures. Genome Res. 2012;22:549-56.

54. Gremme G, Steinbiss S, Kurtz S. GenomeTools: a comprehensive software library for efficient processing of structured genome annotations. IEEE/ACM Trans Comput Biol Bioinform. 2013;10:645-56.

55. Zerbino DR, Birney E. Velvet: Algorithms for de novo short read assembly using de Bruijn graphs. Genome Res. 2008;18:821-9.

56. Boetzer M, Henkel CV, Jansen HJ, Butler D, Pirovano W. Scaffolding preassembled contigs using SSPACE. Bioinformatics. 2011;27:578-9.

57. Boetzer M, Pirovano W. Toward almost closed genomes with GapFiller. Genome Biol. 2012;13:R56.

58. Kumar S, Blaxter ML. Simultaneous genome sequencing of symbionts and their hosts. Symbiosis. 2011;55:119-26.

59. Kumar S, Jones M, Koutsovoulos G, Clarke M, Blaxter M. Blobology: exploring raw genome data for contaminants, symbionts and parasites using taxonannotated GC-coverage plots. Front Genet. 2013;4:237.

60. Camacho C, Coulouris G, Avagyan V, Ma N, Papadopoulos J, Bealer K, et al. BLAST+: architecture and applications. BMC Bioinformatics. 2009;10:421.

61. Buchfink B, Xie C, Huson DH. Fast and sensitive protein alignment using DIAMOND. Nat Methods. 2015;12:59-60.

62. Lee E, Helt GA, Reese JT, Munoz-Torres MC, Childers CP, Buels RM, et al. Web Apollo: a web-based genomic annotation editing platform. Genome Biol. 2013;14:R93.

63. Stanke M, Waack S. Gene prediction with a hidden Markov model and a new intron submodel. Bioinformatics. 2003;19:ii215-25.

64. Jones P, Binns D, Chang H-Y, Fraser M, Li W, McAnulla C, et al. InterProScan 5: genome-scale protein function classification. Bioinformatics. 2014;30:1236-40.

65. Conesa A, Götz S, García-Gómez JM, Terol J, Talón M, Robles M. Blast2GO: a universal tool for annotation, visualization and analysis in functional genomics research. Bioinformatics. 2005;21:3674-6.

66. Bailey TL, Boden M, Buske FA, Frith M, Grant CE, Clementi L, et al. MEME SUITE: tools for motif discovery and searching. Nucleic Acids Res. 2009;37: W202-8.

67. Bolger AM, Lohse M, Usadel B. Trimmomatic: a flexible trimmer for Illumina sequence data. Bioinformatics. 2014;30:2114-20.

68. Kim D, Pertea G, Trapnell C, Pimentel H, Kelley R, Salzberg SL. TopHat2: accurate alignment of transcriptomes in the presence of insertions, deletions and gene fusions. Genome Biol. 2013;14:R36.

69. Quinlan AR, Hall IM. BEDTools: a flexible suite of utilities for comparing genomic features. Bioinformatics. 2010;26:841-2.

70. Li B, Dewey CN. RSEM: accurate transcript quantification from RNA-Seq data with or without a reference genome. BMC Bioinformatics. 2011;12:323.

71. Robinson MD, McCarthy DJ, Smyth GK. edgeR: a Bioconductor package for differential expression analysis of digital gene expression data. Bioinformatics. 2010;26:139-40.

72. Haas BJ, Papanicolaou A, Yassour M, Grabherr M, Blood PD, Bowden J, et al. De novo transcript sequence reconstruction from RNA-seq using the Trinity platform for reference generation and analysis. Nat Protoc. 2013:8:1494-512.

73. Sievers F, Wilm A, Dineen D, Gibson TJ, Karplus K, Li W, et al. Fast, scalable generation of high-quality protein multiple sequence alignments using Clustal Omega. Mol Syst Biol. 2011;7:539.

74. Darriba D, Taboada GL, Doallo R, Posada D. ProtTest 3: fast selection of bestfit models of protein evolution. Bioinformatics. 2011;27:1164-5.

75. Stamatakis A. RAxML version 8: a tool for phylogenetic analysis and postanalysis of large phylogenies. Bioinformatics. 2014;30:1312-3.

76. Finn RD, Clements J, Arndt W, Miller BL, Wheeler TJ, Schreiber F, et al. HMMER web server: 2015 update. Nucleic Acids Res. 2015;43:W30-8.

77. Käll L, Krogh A, Sonnhammer EL. Advantages of combined transmembrane topology and signal peptide prediction—-the Phobius web server. Nucleic Acids Res. 2007;35:W429-32.

78. Li H, Durbin R. Fast and accurate short read alignment with BurrowsWheeler transform. Bioinformatics. 2009;25:1754-60.

79. Garrison E, Marth G. Haplotype-based variant detection from short-read sequencing. Preprint at arXiv 2012:1207.3907v2 [q-bio.GN].

80. Tan A, Abecasis GR, Kang HM. Unified representation of genetic variants. Bioinformatics. 2015;31:2202-4. 
81. Cingolani P, Platts A, Wang LL, Coon M, Nguyen T, Wang L, et al. A program for annotating and predicting the effects of single nucleotide polymorphisms, SnpEff: SNPs in the genome of Drosophila melanogaster strain w1118; iso-2; iso-3. Fly. 2012;6:80-92.

82. Cleary JG, Braithwaite R, Gaastra K, Hilbush BS, Inglis S, Irvine SA, et al. Comparing variant call files for performance benchmarking of nextgeneration sequencing variant calling pipelines. bioRxiv 2015. doi:10.1101/ 023754.

83. Plotree D, Plotgram D. PHYLIP-phylogeny inference package (version 3.2). Cladistics. 1989;5:163-6.

84. Fischer S, Brunk BP, Chen F, Gao X, Harb OS, lodice JB, et al. Using OrthoMCL to assign proteins to OrthoMCL-DB groups or to cluster proteomes into new Ortholog groups. Curr Protoc Bioinformatics. 2011;Chapter 6:Unit 6.12.1-19.

85. Lex A, Gehlenborg N. Points of view: sets and intersections. Nat Methods. 2014;11:779-9

86. Grant CE, Bailey TL, Noble WS. FIMO: scanning for occurrences of a given motif. Bioinformatics. 2011;27:1017-8.

87. de Boer JM, Yan Y, Smant G, Davis EL, Baum TJ. In situ hybridization to messenger RNA in Heterodera glycines. J Nematol. 1998;30:309-12.

88. Lunt DH, Kumar S, Koutsovoulos G, Blaxter ML. The complex hybrid origins of the root knot nematodes revealed through comparative genomics. Peer J. 2014;2, e356.

89. Blaxter ML, De Ley P, Garey JR, Liu LX, Scheldeman P, Vierstraete A, et al. A molecular evolutionary framework for the phylum Nematoda. Nature. 1998; $392: 71-5$

90. Qin L, Kudla U, Roze EH, Goverse A, Popeijus H, Nieuwland J, et al. Plant degradation: a nematode expansin acting on plants. Nature. 2004;427:30.

91. Vanholme B, Haegeman A, Jacob J, Cannoot B, Gheysen G. Arabinogalactan endo-1, 4-galactosidase: a putative plant cell wall-degrading enzyme of plant-parasitic nematodes. Nematology. 2009;11:739-47.

92. Kudla U, Milac AL, Qin L, Overmars H, Roze E, Holterman M, et al. Structural and functional characterization of a novel, host penetration-related pectate lyase from the potato cyst nematode Globodera rostochiensis. Mol Plant Pathol. 2007:8:293-305.

93. De Boer J, Davis E, Hussey R, Popeijus H, Smant G, Baum T. Cloning of a putative pectate lyase gene expressed in the subventral esophageal glands of Heterodera glycines. J Nematol. 2002;34:9.

94. Gao B, Allen R, Maier T, McDermott JP, Davis EL, Baum TJ, et al. Characterisation and developmental expression of a chitinase gene in Heterodera glycines. Int J Parasitol. 2002;32:1293-300.

95. Jones JT, Furlanetto C, Bakker E, Banks B, Blok V, Chen Q, et al. Characterization of a chorismate mutase from the potato cyst nematode Globodera pallida. Mol Plant Pathol. 2003;4:43-50.

96. Bauters L, Haegeman A, Kyndt T, Gheysen G. Analysis of the transcriptome of Hirschmanniella oryzae to explore potential survival strategies and hostnematode interactions. Mol Plant Pathol. 2014;15:352-63.

97. Craig JP, Bekal S, Niblack T, Domier L, Lambert KN. Evidence for horizontally transferred genes involved in the biosynthesis of vitamin B1, B5, and B7 in Heterodera glycines. J Nematol. 2009;41:281.

98. Craig JP, Bekal S, Hudson M, Domier L, Niblack T, Lambert KN. Analysis of a horizontally transferred pathway involved in vitamin B6 biosynthesis from the soybean cyst nematode Heterodera glycines. Mol Biol Evol. 2008;25: 2085-98.

99. Veronico P, Jones J, Di Vito M, De Giorgi C. Horizontal transfer of a bacterial gene involved in polyglutamate biosynthesis to the plant-parasitic nematode Meloidogyne artiellia. Febs Lett. 2001;508:470-4.

100. Scholl EH, Thorne JL, McCarter JP, Bird DM. Horizontally transferred genes in plant-parasitic nematodes: a high-throughput genomic approach. Genome Biol. 2003;4:R39.

101. Paganini J, Campan-Fournier A, Da Rocha M, Gouret P, Pontarotti P, Wajnberg $E$, et al. Contribution of lateral gene transfers to the genome composition and parasitic ability of root-knot nematodes. PLoS One. 2012;: $: 50875$

102. Clifton W, Bird DM, Waterston RH. Analysis and functional classification of transcripts from the nematode Meloidogyne incognita. Genome Biol. 2003;4:R26.

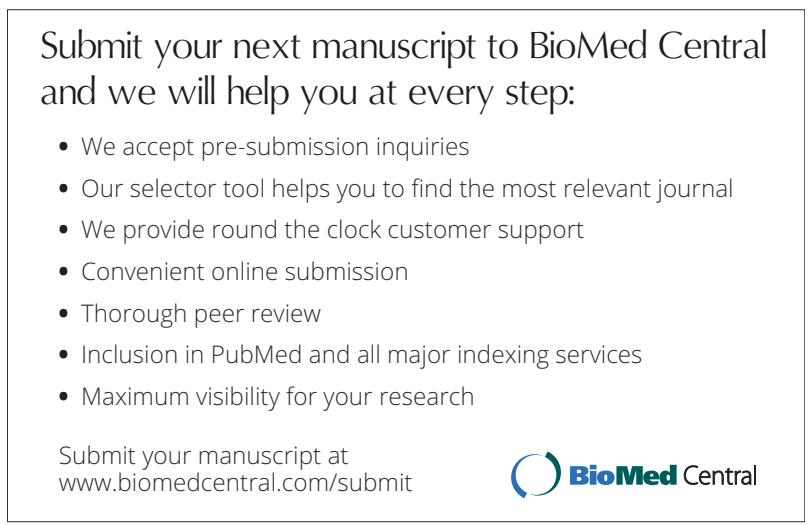

